\title{
Task Transfer by a Developmental Robot
}

\author{
Yilu Zhang, Member, IEEE, and Juyang Weng, Senior Member, IEEE
}

\begin{abstract}
Scaffolding is a process of transferring learned skills to new and more complex tasks through arranged experience in open-ended development. In this paper, we propose a developmental learning architecture that enables a robot to transfer skills acquired in early learning settings to later more complex task settings. We show that a basic mechanism that enables this transfer is sequential priming combined with attention, which is also the driving mechanism for classical conditioning, secondary conditioning, and instrumental conditioning in animal learning. A major challenge of this work is that training and testing must be conducted in the same program operational mode through online, real-time interactions between the agent and the trainers. In contrast with former modeling studies, the proposed architecture does not require the programmer to know the tasks to be learned and the environment is uncontrolled. All possible perceptions and actions, including the actual number of classes, are not available until the programming is finished and the robot starts to learn in the real world. Thus, a predesigned task-specific symbolic representation is not suited for such an open-ended developmental process. Experimental results on a robot are reported in which the trainer shaped the behaviors of the agent interactively, continuously, and incrementally through verbal commands and other sensory signals so that the robot learns new and more complex sensorimotor tasks by transferring sensorimotor skills learned in earlier periods of open-ended development.
\end{abstract}

Index Terms-Attention, classical conditioning, incremental learning, instrumental conditioning, mental architecture, mental development, multitask learning, online learning, scaffolding, skill transfer.

\section{INTRODUCTION}

$\mathbf{T}$ HE WORK reported here is inspired by animal learning models. However, due to the state of knowledge, we do not intend to fit detailed biological data. Although a lot is known about the brain, the knowledge for an agent-wise design is still sparse, especially with regard to development.

Let us consider major existing work on computational modeling of animal learning.

\section{A. Studies on Computational Modeling of Animal Learning}

Classical conditioning [1] and instrumental conditioning [2] are two well-studied models of animal learning. In machine learning, there have been many models that explore their use for

Manuscript received April 24, 2005; revised October 3, 2005. This work was supported in part by the National Science Foundation under Grant IIS 9815191, in part by the Defense Advanced Research Projects Agency (DARPA) ETO under Contract DAAN02-98-C-4025, and in part by DARPA ITO under Grant DABT63-99-1-0014.

Y. Zhang is with the Research and Development Center, General Motors Corporation, Warren, MI 48090 USA (e-mail: yilu.zhang@ gm.com).

J. Weng is with the Department of Computer Science and Engineering, Michigan State University, East Lansing, MI 48824 USA (e-mail: weng@cse.msu.edu).

Color versions of one or more of the figures in this paper are available online at http://ieeexplore.ieee.org.

Digital Object Identifier 10.1109/TEVC.2006.890269 machines [3]-[6]. See [7] for an excellent survey. Some of these models have been implemented on robots. For example, in [8] and [9] a robot functioned upon a set of percepts and behaviors that were defined in advance. In [10], a mobile robot learned to associate the visual sensations of predefined classes (blob and stripe patterns on cubes) with predefined action sequences.

These studies have greatly advanced computational understanding of animal learning. They are meant for situations where the human programmer is given a task so that he can design part of the agent's internal representation, including feature detectors and internal states. For example, the type of features are predetermined from the known environmental conditions. Alternatively, the task to be learned is known so that one can define internal variables such as the symbol "US-food" as a type of unconditional stimulus and the symbol "UR-salivation" for a type of unconditional response. Indeed, a major characteristic of traditional AI or recognition systems is that the implementation starts from a given task, so that internal symbolic representation is possible. Different learning strategies and algorithms have developed after the human designer analyzes the task specifically. In other words, traditional man-made systems depend largely on human intelligence in designing task-level symbolic representation.

Both natural and artificial intelligences face a fundamental challenge in understanding and modeling development in which an agent (human or robot) must learn tasks that were not necessarily predictable at birth (for humans) or during the programming time (for machines), to such a full degree that the genome or hand-design is sufficient for storing full task-specific representation [11], [12]. For example, in traditional automatic speech recognition by a computer, speech streams for training are manually segmented by humans into linguistic units (e.g., words) so that a particular hidden Markov model (HMM) that is hand-designed for this word receives only samples of a single word during training.

Task transfer, by definition, is the transfer of learned skills to new and different task contexts. In the work reported here, we investigated task transfer through development in which the tasks are unknown during the programming time, analogous to human learning where the tasks that a baby will end up learning are not fully predictable at birth. All of the tasks, to be learned or to be executed, are invoked by the corresponding contexts, during an open-ended learning process. The contexts are either provided by the environment (including human teachers) or the agent itself. By open-ended learning, in this paper, we mean that the system must maintain real-time speed while the memory grows large (i.e., logarithmic time complexity); the tasks to be learned are unknown during the programming time, but are restricted to sensorimotor skills only.

In such a developmental process, in contrast with the traditional work, no symbolic internal representation is applicable. 
The internal representation are self-generated and self-organized vectors, representing clusters similar to neural response patterns. The learning takes place while the agent interacts with the unknown environment by sensing real-time sensory streams directly. For example, the number of speakers, the number of words and phrases, the required association between the sensory inputs and behaviors, and even the tasks that are to be learned are unknown during the programming time. The required internal self-organization is very challenging.

\section{B. Representation and Architecture}

For this type of open ended learning, symbolic representations is not suited for internal representation for two major reasons. (1) The meaning of every symbol is understood by the programmer. However, he is not available to call up the correct symbol (e.g., a node in a Markov model) to link with the dynamically occurring environmental event so that the sensory event is attributed correctly to the correct element of internal representation. (2) The static hand-designed symbolic representation as a whole is not sufficient for an open-ended number of tasks. For example, a fixed set of nodes in a HMM cannot adapt well to the increasing and changing degrees of internal freedom required by an open-ended number of tasks learned through development.

We desire to synthesize an architecture with internally varying degrees of freedom to deal with learning directly from sensory streams. Unlike a major goal in psychology which aims to analyze different types of animal learning using different models (e.g., classical conditioning and instrumental conditioning) [5], [13], [14], the goal here is to synthesize a single architecture that incorporates various learning modes including classical conditioning, instrumental conditioning, task transfer, and so on, to learn an open-ended number of unknown tasks without using any internal symbolic representation. This goal is, of course, too challenging to be addressed in its full scale here, since there are many puzzles of the brain that are unknown. Therefore, we restrict ourselves only to sensorimotor skills that do not require an extended high-level thinking process. For example, the temporal length of the sensory context vector is limited to around $2 \mathrm{~s}$.

\section{Architecture Challenges}

However, even the sensorimotor skill development is very challenging for this type of open-ended learning, since we must enable machines to learn directly from raw sensory streams for unknown tasks.

In the psychological and robotics literature, classical conditioning (without reinforcer) [1], [5], [7], instrumental conditioning (with positive or negative reinforcer) [6], [9], [15], [16], and extinction (absence of expected reward) [1], [17]-[19] are described by different learning models, although there have been studies that investigate the effect of reinforcer on classical conditioning, e.g., [20]. These models do not explain why and how a single complex brain architecture displays such a wide variety of learning types.

In an open-ended sensorimotor development, the system does not associate labels with actions, since no label is available from widely varying, raw, numeric sensory inputs. Without a symbol to supervise their grouping, how can different utterances from different speakers of auditory streams be correctly associated with the desired behaviors?

A human does not speak the same way every time. The learner does not know how many classes of verbal utterances are available, and the number of classes increases through development without a definite bound. How does the learnerknow which utterances belong to the same type without even knowing how many types there are? Furthermore, the utterance is not temporally segmented in normal human learning. Where does a meaningful utterance start and where does it end in an open-ended sensory stream? Without such information to guide the temporal segmentation, how can the learner break the endless sensory stream into meaningful segments so that each segment will be linked to a desired behavior? If different segments are segmented incorrectly, how does the learner measure the similarity of different segments? If a particular utterance is associated with a desired action, how can other similar utterances be associated with the same desired action without excessive amounts of explicit learning? Such difficulties were addressed by Zhang et al., where many-to-many mapping, from multiple sensory inputs to multiple patters of internal representation, is reliably established dynamically in real-time. Further, many-to-one mapping, from multiple patterns of internal representation to a single correct action, is established dynamically in real-time, through supervised learning or reinforcement learning. In the work presented here, such difficulties are further dealt with when learned sensorimotor skills must be used in new contexts for new tasks.

As the focus of this paper, let us consider task transfer, which is the capability of autonomously applying what has been learned in one context to new contexts (or new tasks) [22]. Task transfer is more than memorizing and learning. Here is an example. Suppose that a tiger in a circus has learned two skills: (1) Jump onto a table top immediately after hearing the command "Table!" from the trainer. (2) Jump through a fire ring immediately after hearing a "Ring!" command. The new task is to perform (1) and (2) in sequence after hearing a new command, "Start!," without the stepwise commands of "Table!" and "Ring!" As far as we know, there has been no other published work that deals with task transfer in the context of open-ended development.

There are many challenges in this new type of learning, from concept, to theory, to architecture, to algorithm, to implementation. At this early stage of research, the goal of the work reported here is not to achieve the recognition rate or vocabulary size comparable to the best speech recognition systems using the traditional training methods. Instead, the goal is to explore concepts, theory, architecture, algorithms, implementation, and experiments for developmental robots.

In the following sections, we first describe the new concepts, theory, the design principles and the major challenges for the implementation. A single-level learning architecture is presented in Section III followed by a multilevel architecture in Section IV. The latter is crucial for "abstraction" from a wide variation of signals in the real world. Experiments on a real-time system and the SAIL robot are reported in Sections V and VI, respectively. Conclusions are drawn in Section VII. 


\section{CONCEPTS AND THEORY}

Symbolically, classical conditioning has been described as the following so-called acquisition procedure (A.P.):

$$
\mathrm{CS} \rightarrow \mathrm{US} \rightarrow \mathrm{UR} \Rightarrow \mathrm{CS} \rightarrow \mathrm{CR}
$$

where CS stands for conditioned stimuli (e.g., tone), US for unconditioned stimuli (e.g., food), UR for unconditioned response (e.g., salivation), CR for conditioned response (e.g., salivation), $\rightarrow$ means "followed by," and $\Rightarrow$ means "develops." Other conditioning protocols that have been widely studied include secondary conditioning, where one CS is preceded by another CS

$$
\mathrm{CS}_{2} \rightarrow \mathrm{CS}_{1} \rightarrow \mathrm{CR} \Rightarrow \mathrm{CS}_{2} \rightarrow \mathrm{CR}
$$

and instrumental conditioning, where the association between a stimulus $(S)$ and a response $(R)$ is affected by the following positive or negative reinforcement (reward).

These models have been very useful for understanding animal learning. However, they are not meant to describe how the brain deals with these and other different types of learning without being told explicitly what type of learning is in the mind of the teacher. If the type of learning were revealed to the brain explicitly by some magic force before the learning takes place, the brain could have used a dedicated part of itself to deal with this particular type of learning. However, there is no evidence for such a magic force that tells the brain whether the next learning experience will be classical conditioning, instrumental conditioning, task transfer, etc. The brain must develop to deal with all types of learning using a single, highly complex architecture.

Further, these models tend to characterize some specific aspects of a very complex process. This is fine for the purpose of a focused study, but many other aspects must be incorporated if one must build a developmental system that can demonstrate certain capabilities, even limited ones, of development and learning in largely unknown environments, while being faced with learning unknown tasks. For example, one such aspect is selective attention. Traditionally, task-related information is manually extracted for symbolic description as in A.P. (1) and (2), without modeling why only this information is relevant among many other environmental stimuli and bodily internal signals (e.g., motor signals).

\section{A. A Unified Sensorimotor Model}

We do not intend to propose an architecture that can explain all the details of the central nervous system; that is impossible at this state of knowledge. The computational modeling is not meant to be biologically proven. Instead, we intend to design a unified architecture that can explain some major types of learning that are well known in psychology, neural science, and machine learning. We relate the discussion with biological computation in a loose way, wherever appropriate, hoping that such modeling can, in the future, be biologically proved or disproved.

According to Vygotsky's theory of zone of proximal development (ZPD) [23], human teachers typically "arrange experience," rather than teaching didactically. The ZPD proposed by Vygotsky is a latent learning gap between what a child can

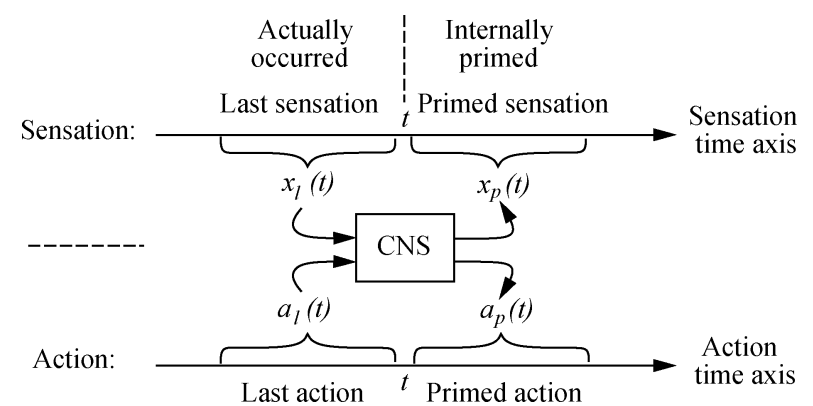

Fig. 1. A developmental agent is a real-time predictor. At any time, the agent has four types of context. It maps the last context (vector) $l(t)=\left(x_{l}(t), a_{l}(t)\right)$ to the selected primed (context) vector $p(t)=\left(x_{p}(t), a_{p}(t)\right)$ from multiple possible primed contexts.

do on his or her own and what can be done with the help of a teacher. Arranged experience, as explained in the above tiger training example, enables the learner to establish desired associations. Later, Wood et al. [24] used the term "scaffolding" to describe such an instructional support through which a child can extend or construct current skills to higher levels of competence. Through this process, the scaffolding (arranged experience) is slowly removed. The tiger in the above example eventually is able to perform a very complex sequence of performance in a circus from a "Start" command of the trainer, without further assistance from the trainer.

Weng [25] proposed that a major computational function of the central nervous system is to perform real-time prediction (or priming), as shown in Fig. 1.

At each time instant $t$, the attended working memory stores the last context, which is a high-dimensional vector (i.e., a response pattern of the cortex)

$$
l(t)=\left(x_{l}(t), a_{l}(t)\right)
$$

which includes the last sensation vector $x_{l}(t)$ at time $t$ and the last action vector $a_{l}(t)$ at time $t$, both represent response patterns of the related cortex. From the last context, the sensorimotor system predicts the primed context

$$
p(t)=\left(x_{p}(t), a_{p}(t)\right)
$$

which consists of the primed sensation vector $x_{p}(t)$ and the primed action vector $a_{p}(t)$. To take into account instrumental conditioning, different regions of the brain provide multiple different primed contexts

$$
\left\{p_{1}(t), \ldots, p_{k}(t)\right\}=R(l(t))
$$

where $R$ is a multivector regression function, carried out by the central nervous system. The value function $V$ in sensorimotor system selects one desired context $p_{i}(t)$ at the current time $t$

$$
V\left(\left\{p_{1}(t), p_{2}(t), \ldots, p_{k}(t)\right\}\right)=p_{i}(t) .
$$

However, this real-time prediction model of a sensorimotor system is not sufficient to take into account internal loops in the sensorimotor system that are required to realize various learning types, including the internal autonomous "thinking" process, e.g., thinking about "food" when hearing a familiar "paired" 


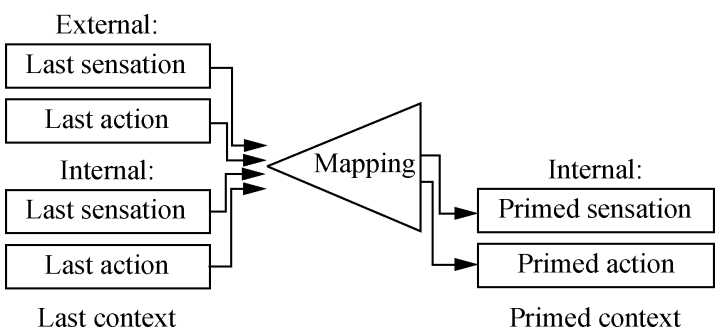

Fig. 2. The mapping of the sensorimotor system enables various types of autonomous reasoning depending on which source the mapping selects as the attended last context.

tone during classical conditioning. Weng [25], [26] proposed an agent model called self-aware self-effecting (SASE) to take into account the need to perceive internal brain activities (e.g., predicted sensation) and the need to affect internal brain activities (e.g., selective attention). With this agent model, the term "internal" refers to the sensorimotor system and the term "external" means outside of the sensorimotor system. To facilitate understanding, the SASE model will be used in this work, but the model itself is not a part of the work reported here.

The regressor, $R$, denoted as a mapping realized by the sensorimotor, takes as input two types of last context: internal (from within sensorimotor) and external (from outside sensorimotor, i.e., the agent body and other parts of the environment), as illustrated in Fig. 2. The mapping includes an attention selector $T$ performed by earlier sensory cortex and the regressor $R$ performed by later association cortex. The intermodal attention by $T$ determines whether the corresponding sensorimotor association in the reasoning process is external, internal, or mixed (both internal and external are taken into account at the same time).

This SASE agent model is general, with the potential of being able to model sensorimotor processes and high-level processes such as autonomous reasoning and autonomous planning, see [27, pp. 157-158]. However, higher level reasoning processes are beyond the scope of this work. We concentrate on task transfer here.

Fig. 3 explains why classical conditioning and instrumental conditioning are special cases of the general prediction function of the sensorimotor system.

Each circle in Fig. 3 denotes a set of many context vectors (response patterns) at a particular discrete time $t_{i}, i=1,2, \ldots$. Two cycles aligned vertically denote two sets of contexts, sensation and action, respectively, at the same time. Observed temporal association is between a particular context vector in one set with another particular context vector in the other set. As shown in the figure, repeated pairings between the last context and the primed context not only establish reliable short-time-separation (or primary) associations denoted by solid lines in the figure, but also long-time-separation (or secondary) associations denoted by the dashed lines. Such long-time-separation associations are always from left to right (time separation), but they can start from either the upper row (attend to sensation) or the lower row (attend to action) and end at either the upper row (predicted sensation), the lower row (recall action), or both (mixed sensation and action as predicted context). The challenging issue of cortical generalization is to generalize from vector-to-vector association to reliable set-to-set association while the set is not explicitly given.

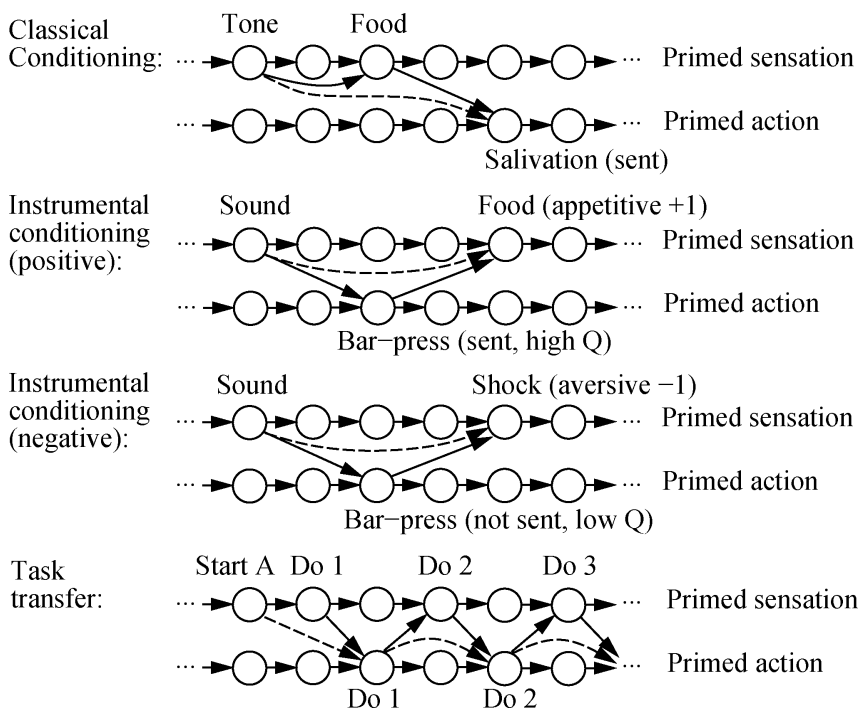

Fig. 3. Context prediction of the sensorimotor system enables classical conditioning, instrumental conditioning, and task transfer. Each circle in the figure indicates a set of many corresponding states (clustering vectors, i.e., similar neural response patterns), represented by the best matched ("resonate") contexts in the long-term memory. From left to right is the passing time. Solid arrows denote short-time-separation associations between a set of vectors to another set of vectors. Dashed arrows denote autonomously learned association through time.

In fact, the situation of nonsymbolic representation is more challenging than what is plotted in Fig. 3. The vectors do not cluster within individual cycles as shown for simplicity, but rather, they spread out continuously in the sensory space, as shown in Fig. 4(a). IHDR [28], [29] ${ }^{1}$ is used to automatically divide the high-dimensional regions into many small regions, while disregarding irrelevant dimensions. Every small region is represented by a few microclusters, as shown in Fig. 4(b). The underlying developmental mechanisms will be explained in Section II-E.

\section{B. Task Transfer}

Task transfer is achieved through communicative learning, as proposed in [25], which includes two phases: 1) language (or sensorimotor-skill) acquisition and 2) learning using language (or using sensorimotor skills), i.e., transfer.

In the sensorimotor-skill acquisition phase, the SAIL robot learned to associate last context $l_{i}$ with the primed context $p_{i}$, $i=1,2, \ldots, n$, denoted as

$$
l_{i} \rightarrow p_{i}
$$

They are called basic skills. In the learning using sensorimotorskill phase, the basic skills are transferred to new settings for more complex sensorimotor skills. A new context $l^{\prime}$ is the environmental cue to trigger a sequence of desired sensorimotor events

$$
l^{\prime} \rightarrow p_{1} \rightarrow p_{2} \rightarrow \cdots \rightarrow p_{n}
$$

without a hint cue $l_{i}, i=1,2, \ldots, n$ at the right time. In other words, the basic sensorimotor skills learned in the early phases indicated by (7) cannot simply be copied without any change.

${ }^{1}$ IHDR stands for incremental hierarchical discriminant regression. A brief discussion on IHDR is given in Section II-E. 


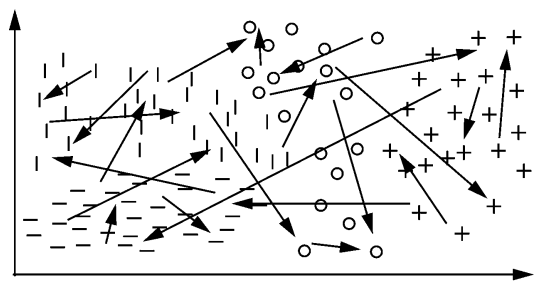

(a)

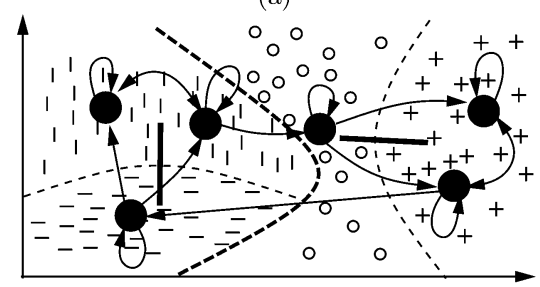

(b)

Fig. 4. Automatically sort out a temporal mess for superior local invariance. A sign,,$- \mid, \circ$, or + , indicates an input sample $(x, y)$, whose position indicates the position of $x$ in the input space and whose sign type indicates the corresponding $y$ output. An arrow indicates consecutive firing between two time steps. (a) A mess of temporal transitions: A 2-D illustration of many-to-many temporal trajectories of samples (consecutively firing neuron patterns) in the original high-dimensional continuous sensory space. (b) Cleaner transitions between fewer primitive prototypes (black circles), enabled by IHDR. A solid line indicates a most discriminating subspace of IHDR with a significantly reduced dimension, illustrated as 1-D. A solid black cycle indicates a primitive prototype in the leaf node of IHDR. The samples indicated by the signs,,$- \mid, \circ$, and + are not kept by the IHDR tree.

Since the contexts are different, the basic skills must be used as building blocks to build more complex tasks indicated by (8). In the new complex task, the basic skills have also been modified (i.e., transferred) so that they are used for the new contexts, e.g., $p_{i}$ must be associated with $p_{i-1}$ instead of the original $l_{i}, i=$ $2,3, \ldots, n$.

\section{Required Mechanisms}

Let us consider an example that is concrete but still general enough to see the underlying mechanism and the general power of task transfer. The previous acquired skills are the coupling of receiving the (verbal) command $C_{i}$ with action output $A_{i}$

$$
C_{i} \rightarrow A_{i}, i=1,2,3,4
$$

The (verbal) command is spoken in a natural setting and may be produced by different speakers. The action $A_{i}$ is the desired motor sequence in response to $C_{i}, i=1,2,3,4$. In this sensorimotor-skill acquisition phase, any type of learning can be used. In our experiment, this is done by stating a verbal command and then guiding the compliant robot arm (through microswitches) to perform the corresponding action. $C_{1}, C_{2}, C_{3}$, and $C_{4}$ correspond to auditory inputs "upper," "lower," "left," and "right," respectively. $A_{1}, A_{2}, A_{3}$, and $A_{4}$ correspond to the desired motor sequences to draw the upper, lower, left, and right petals of a flower, respectively. This is a process of acquisition of sensorimotor skills in an embodied, incremental and real-time fashion.

With the same developmental program continuously learning, the next session implicitly in the mind of the human teacher is to use the acquired language (or sensorimotor) skills to learn more complex sensorimotor behaviors. For example, the teacher wants to give a new command, e.g., $C$, and, he desires that the robot obey by executing actions $A_{1}, A_{2}, A_{3}, A_{4}$, consecutively, and then stopping. However, the robot has only learned to respond to command $C_{i}$ with action $A_{i}$ and at the end of action $A_{i}$, it stops.

The key in making the transfer possible is to bring the desired contexts into a close temporal neighborhood, i.e., scaffolding as analyzed by Vygotsky [23]. This is a typical teaching method carried out by providing a hint at the right time and letting the priming mechanism work. For example, when the teacher states $C$, which the machine does not understand and invokes no action, the teacher next states the verbal command $C_{1}$ immediately so that the verbal command brings the associated action $A_{1}$ into close temporal proximity of $C$, as formulated below

$$
C \rightarrow C_{1} \rightarrow A_{1}
$$

The priming mechanism of Q-learning [30], through the prototype updating queue in our architecture to be explained later, backpropagates the context $A_{1}$ through state-transition traces so that the prototype of the context $C$ primes not only the sensory context $C_{1}$ but also action $A_{1}$, denoted as

$$
C \rightarrow\left\{C_{1}, A_{1}\right\}
$$

where $\left\{C_{1}, A_{1}\right\}$ indicates that both the sensory context and the action context are primed. In other words, after a few practices, the agent will recall sensation $C_{1}$ and action $A_{1}$ as soon as it receives sensory context $C$. According to the operation of the architecture, the action $A_{1}$ is executed. This developmental learning process is denoted as

$$
C \rightarrow C_{1} \rightarrow A_{1} \Rightarrow C \rightarrow A_{1}
$$

This is the second phase of communicative learning: learning using acquired sensorimotor skills. This type of learning is typically more efficient than exploration since the desired action $A_{1}$ is hinted directly by the human teacher, instead of based on trying all possible actions using, e.g., Boltzmann exploration. For example, when knowledge has been discovered, learning from a seminar is more efficient than rediscovery through exploration. Although only sensorimotor skills are involved here, the principle is the same.

Furthermore, this is not the traditional supervised learning in the following sense: The information about the desired output is not fed through the effector channel, but instead through the regular sensory channel, which can contain any other environmental information.

To build the later actions, the tail context of $A_{1}$ serves a similar role as $C$ if the teacher states verbal command $C_{2}$ as soon as $A_{1}$ ends. Analogously, after a few practices, we have

$$
A_{1} \rightarrow C_{2} \rightarrow A_{2} \Rightarrow A_{1} \rightarrow A_{2} \text {. }
$$

An important mechanism that is needed for the success of such learning is the intermodal attention mechanism: attend to $C_{1}$ or $A_{1}$ in (9), as illustrated in Fig. 2. The value system for intermodal attention behavior is such that more novel context is attended in the absence of aversive and appetitive stimuli [31]. $A_{1}$ is more novel than $C_{1}$ since its sequence ended later than $C_{1}$. 


\section{Generality of the Sensorimotor Prediction Model}

It is important to note that the symbols in the above model are not symbols in the internal representation. Each context corresponds to one of the many possible patterns of responses of the related cortex. There is no symbol at all in our biologically motivated internal representation. All the representations are numeric vectors, namely, patterns of cortical responses. That is why the agent is able to develop its more complex sensorimotor skills from simpler sensorimotor skills that are learned early. Such a developmental process involves not only interactions with the environment (including teachers) but also the active participation of the agent.

From the above discussion, we can see that although different types of learning may involve very different areas of the brain, classical conditioning, instrumental conditioning, and task transfer share the same computational nature: prediction of a context from the last context as illustrated in Fig. 3.

Classical conditioning predicts an action from attended external sensation. Instrumental conditioning predicts the sensation from the attended last sensation and last action and then uses the value system to select the attended action depending on the estimated value of the predicted sensation. Task transfer is a multistage prediction of primed context from attended last context. Of course, task transfer can evolve the value system so that certain associations can be encouraged (using reward) or discouraged (using punishment). Therefore, classical conditioning, instrumental conditioning, and task transfer can all be described by the prediction model in Fig. 1, if its sensorimotor system has an attention selection, as shown in Fig. 2 and a value system defined in (6).

If an architecture can implement the prediction of primed contexts from the last context and use value to select a primed context, the resulting developmental program can continuously run to acquire more and more sensorimotor skills without the need to redesign the program, even though the tasks that the agent ends up learning in the future are unknown to the programmer. Of course, in practice, there exist many technical challenges in implementing this theoretical model by a machine.

\section{E. Technical Challenges and Developmental Mechanisms}

To enable open-ended scaffolding through development, the training and test phases must be conducted in the same operational mode through online real-time interactions between the robot and the environment (including the teacher).

The technical challenges for achieving task transfer are numerous. To be self-contained, we first summarize the challenges that have been addressed in our prior work, which is followed by the major challenge and resolutions specifically reported in this paper.

1) Continuous High-Dimensional Sensory Space: Since we deal with raw sensory streams, the dimension of $l(t)$ is as high as hundreds or thousands, if audition or vision is involved, respectively. Further, the number of possible vectors of last context $l(t)$ is potentially infinite. The space of all possible last contexts $l(t)$ is

$$
\mathcal{L}=\{l(t) \mid t=0,1,2, \ldots\}
$$

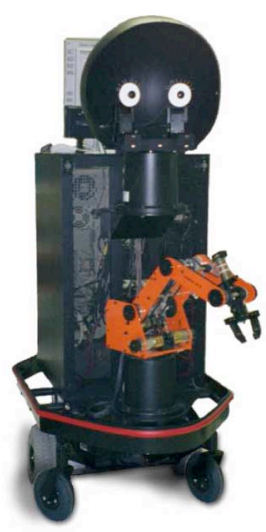

(I)

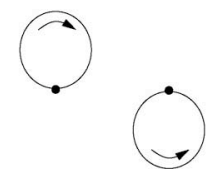

(a)

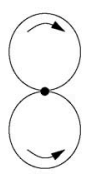

(e) (b)

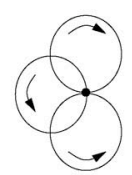

(f) (c)

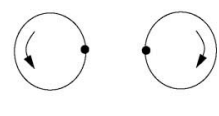

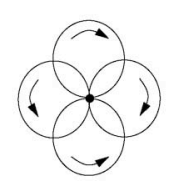

(g)
(II)

Fig. 5. (I) The SAIL robot house built at Michigan State University (II) Behaviors represented as gripper tip trajectories of the SAIL robot. (a)-(d) Basic skills as petal drawing, each of which starts from the black dot. (e)-(g) Drawings consists of more than one petal, as skills developed through multiple task transfers.

Much of the space is, however, never sensed. For example, the vectors of the last context must correspond to natural sounds that humans typically hear. We need a developmental mechanism that automatically finds the manifolds in which the input data lie, for both efficiency of representation (small cortical regions) and better generalization from fewer samples.

We model self-organization in the cortex using IHDR [28], [29], as illustrated in Fig. 6.

Each node in the IHDR tree corresponds to a cortical region. The space $\mathcal{L}$ of last contexts $l$, represented as $d$-dimensional vectors, is first partitioned coarsely by the root of the IHDR tree. The root partitions the space $\mathcal{L}$ into $q$ subregions $(q=4$ in the figure), each corresponds to one of its $q$ children. Each child partitions its own smaller region into $q$ subregions again. Such a coarse-to-fine partition recursively divides input space into increasingly small input regions through the developmental experience. The $q$ neurons (called $x$-clusters) in each node corresponds to a set of feature detectors. With incrementally arriving input vectors $l(t)$, these $q$ neurons perform competitive incremental updates, until they are mature enough to spawn children, determined by the number of samples received by the node.

The incremental self-organization in each node uses a variant of the well-known statistical technique called the linear discriminant analysis (LDA), so that the $q$ neurons in each node detect the most discriminant features (MDFs). By most discriminating, we mean that these features "best explain" the betweenclass variance in the input space (region) of the node, where the class ID are incrementally formed clusters in the output space. LDA implies that all input components that are irrelevant to output (e.g., background pixels in face images where IDs are person IDs) are automatically disregarded for better generalization using a small tree (cortical areas). This requires that $x$-clusters are formed according to class-labels, but numeric outputs do not provide explicit class labels.

To resolve this missing-label problem, IHDR incrementally forms $q$ clusters, called $y$-clusters, in the output space for each internal node. The $y$-clusters are formed according to the $y$-part of the teaching $\left(x_{i}, y_{i}\right)$ pairs. The virtual label of $y_{i}$ according 


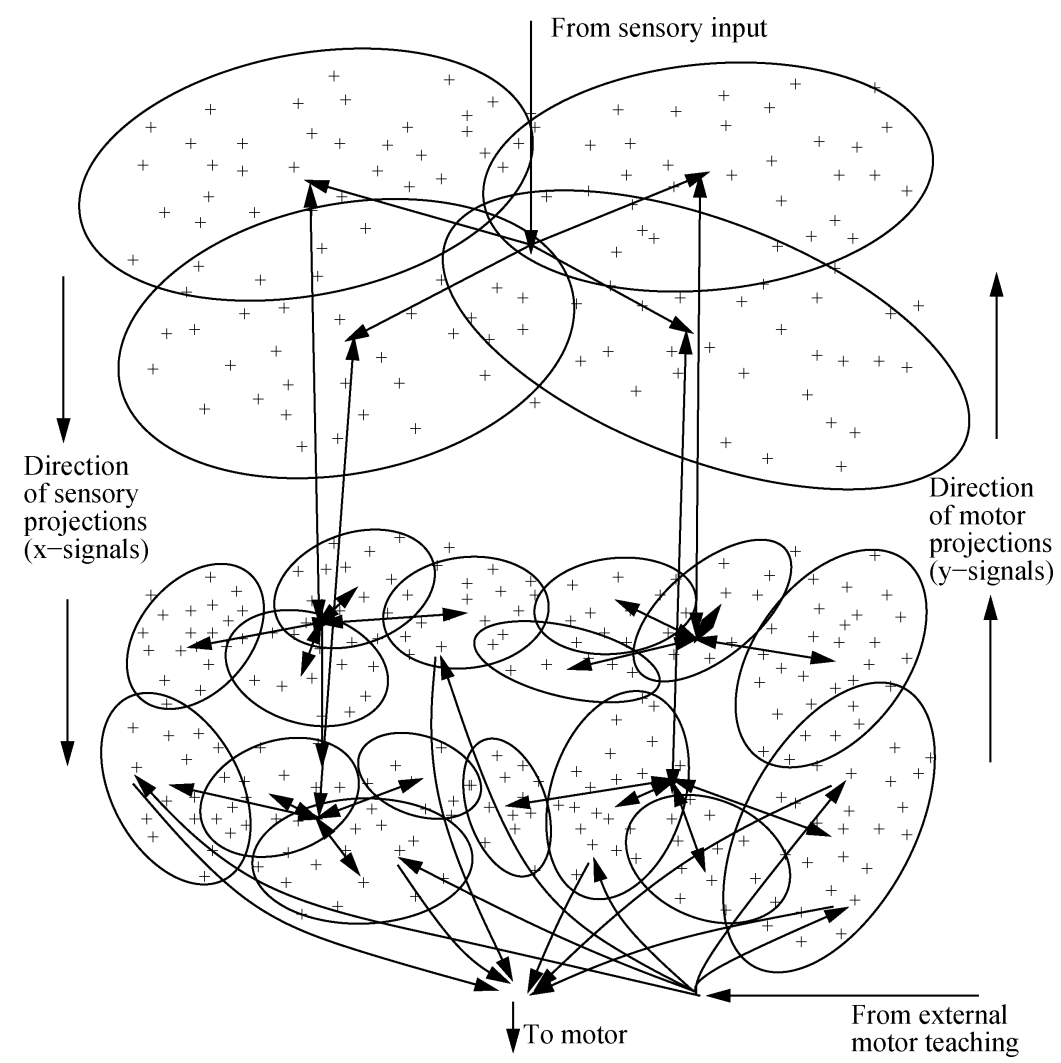

Fig. 6. Illustration of an IHDR tree incrementally developed (self-organized) from experience. The sensory space is represented by sensory input vectors, denoted by "+." The sensory space is repeatedly partitioned in a coarse-to-fine way. The upper level and lower level represent the same sensory space, but the lower level partitions the space finer than the upper level does. An ellipse indicates the space that the neuron at the center is responsible for. Each node (cortical region) has $q$ feature detectors (neurons) ( $q=4$ in the figure), which collectively determine which child node (cortical region) the current sensory input belongs to (excites). An arrow indicates a possible path of the signal flow. In every leaf node, prototypes (marked by "+") are kept, each of which is associated with the desired motor output.

to the nearest $y$-cluster, provides the class label information for the corresponding $x_{i}$ to perform supervised (labeled) clustering. Therefore, the $q-1$ dimensional hyperplane that passes the centers of the $x$-clusters are the MDF feature space. Each input vector is projected onto this $(q-1)$-dimensional feature subspace in each node. That is why the clusters are presented as points along a 1-D line (MDF feature subspace) in Fig. 4(b). The direction orthogonal to the solid lines are disregarded for better generalization. In practice, typically $q \ll d$.

In the MDF subspace, statistically optimal weights are automatically updated (developed) for every MDF, according to $(q-1)$-dimensional within-class correlation matrices, so that the partition boundaries resulting from $q$ feature detectors are cut along nonlinear class boundaries as much as possible, which further leads to better generalization using a small tree (smaller cortical areas). Illustrated graphically, the solid lines in Fig. 4(b) point to directions along which the corresponding motor action greatly changes. The piecewise dashed lines form nonlinear decision boundaries. Different nodes in the same level collectively tessillate the nonlinear manifold represented by the sensed region of input space, using an automatically determined number of cells.

The leaf nodes in the tree (i.e., nodes without children) are nodes that have not received enough input samples to attain maturity. Each leaf node keeps $m>q$ microclusters, which are incrementally created primitive prototypes of $l(t)$ received from its parent node. Each microcluster $l^{\prime}$ is the incremental average of input vectors $l(t)$ that the microcluster wins during similarity-based competition among $m$ microclusters. Each microcluster $l^{\prime}$ (neuron) is associated with an output vector $a(t)$, which is the incremental average of all the action vectors associated with input vectors $l(t)$ that $l^{\prime}$ won over. Therefore, the continuous input space $\mathcal{L}$ and the continuous output space $\mathcal{P}$ are represented by a hierarchical, discrete number of neurons represented as an IHDR tree (a pathway of cortical areas). Internally, there is not a monolithic symbolic representation, but different internal representations may lead to the same external action.

The IHDR tree, a computational model of related artificial cortical areas, is grown gradually by being updated (developing) incrementally with one input vector at a time. During the action imposed learning, the motor signal imposed by the environment (teacher) is also used. Given any last context vector $l(t)$, the coarse-to-fine search through the IHDR tree reaches the "best matched" microcluster $l^{\prime}$ (premotor neuron) which is associated with the desired action $a$ (motor neuron). However, in general, the output vectors associated with $l^{\prime}$ are associated with multiple primed contexts (neurons) $\left\{p_{1}, p 2, \ldots, p_{k}\right\}$, as indicated in (5). In summary, IHDR incrementally and quasi-optimally approximates and generalizes observed associations from arriving sensory inputs. IDHR outperformed feedforward networks, radial basis functions, and the support vector machines (SVMs) in our studies. For further detail, the reader is referred to [28] and [29]. 
The capability of IHDR is critical for the success of the architecture reported here. The feedforward networks with backpropagation learning and radial basis functions with iterative adaptation are not suited for the demanding requirements here, mainly because of their fixed degrees of freedom. The SVM is not suited either because it is a batch, computationally slow learning method.

2) Missing the Number of Classes: It is not known how many meaningful classes exist in the input space. The output is continuous motor space $\mathcal{P}=\{a(t) \mid t=0,1,2, \ldots\}$. Similar to cortical representation, the number of microclusters in the IHDR tree is much larger than the number of classes. As long as each input vector $l(t)$ is mapped to the desired motor action $a(t)$, there is no need to figure out exactly how many classes there are in $\mathcal{L}$. Unlike the prior work that uses a symbolic representation, different context vectors that belong to the same class (e.g., US-food) are matched to multiple microclusters (premotor neurons), instead of a single symbol.

3) Scalable to Large Memory But Real-Time Speed: The number of microclusters in the sensory space corresponds to part of the long-term memory, which can grow very large. The absolute amount of disk storage of the hard disks is not a major problem. The major scalability problems are: 1) good performance and 2) real-time speed. Both are maintained when the memory size is very large. Due to the dynamically spawning nodes and self-organization through competition, the terrain of the input is dynamically covered by the limited resource based on experience (utility). The performance is scalable since there is no overfitting or underfitting by a method of a fixed degree-of-freedom (e.g., backpropagation learning using a multilayer feedforward network). The scalability with real-time is guaranteed by the logarithmic time complexity $O(d \log (n))$, where $n$ is the number of nodes in the IHDR tree.

Building upon the IHDR technology, the work reported here addresses the following challenges in order to accomplish task transfer through development.

4) Missing Context: The discussions in Section II-B illustrate that task transfer requires a prediction mechanism to handle missing follow-up contexts. For example, on the right side of A.P. (10), the context $C$ has to elicit $A_{1}$ without the presence of the context $C_{1}$. The well-known Q-learning algorithm [30] realizes a mechanism to predict the delayed return by backpropagating, for each state-action pair $(s, a)$, a time discounted reward value through the trajectory of consecutive occurred states. We extended this backpropagation idea. In our model, not only the reward but also the primed context is backpropagated, thanks to the numeric representation. The states in the Q-learning algorithm correspond to the dynamically created and updated microclusters $l^{\prime}$ in the space of last context $\mathcal{L}$ in (11). As a result, not only the return but also the sensation and the action are predicted. The following mechanisms are proposed and experimented with to address the missing context problem collectively. (1) Double tree mappings (R-tree and P-tree) for immediate and near-future priming (Section III-B). (2) A gating system to select the appropriate action to fire (Section III-C). (3) Intermodal attention to determine the desired part of the last context that actively participate in the sensorimotor association (Section III-D).
5) Abstraction: While the numeric representation is more powerful than symbolic representation in describing the necessary details in the sensorimotor association, the challenge is to generalize the sensorimotor associations without tedious prototype-by-prototype learning. For example, a successful prediction from a particular context vector will not be automatically translated into a successful prediction from other similar context vectors. We use a hierarchy of representation with different granularities to reach gradual soft "abstraction." The higher level sensorimotor system has coarser granularity both in space and in time, and the granularity is not necessarily uniform in space and time, as discussed in Section IV.

\section{Single-LeVEl ARChiteCture}

In order to understand the architecture in detail, it is necessary to mathematically formulate a developmental agent.

\section{A. An Agent Model}

By definition, an agent is something that senses and acts. Suppose that the agent is born at time $t=0$ and works in discrete times. The history at time $t$ is defined as $h(t)=\{(x(\tau), a(\tau)) \mid \tau=0,1,2, \ldots, t\}$, which is a realization of the random process from the "birth" time, where $x(\tau)$ is the numerical sensory vector sensed by the agent at time $\tau$, and $a(\tau)$ is the numerical control vector sent by the agent to its effectors at time $\tau$.

The mathematical tool of random process is very useful to model time-varying signals in the brain even if the signals are noise-free and not random in nature. As long as the full reasons (e.g., in the physical world) that give rise to the signals are not practical to be fully modeled (e.g., the physical reasons are unknown), a random process model enables the brain to estimate its distribution using neurons (e.g., each neuron representing a sensed region of the distribution), as well as other statistical properties such as the mean of the distribution. In other words, randomness in a computational model corresponds to a degree of detail in modeling.

The history includes all of the information available to the agent from the "birth" time. The last context at time $t$ is the recent part of history $l(t)=\{(x(\tau), a(\tau)) \mid \tau=t-b(t), \ldots, t-$ $1, t\}$. It consists of a short segment of recent experiences of temporal length $b(t)+1$. Currently in our work, $b(t)$ does not change with $t$ and a different $b(t)$ is designed for different sensors and levels. It corresponds to part of working memory.

A state $s(t)$ is the internal representation of the last context $l(t)$ in an agent's internal information environment. It corresponds to the best matched prototype from the long-term memory.

The agent views the world as a general random process called history. The state gives a more compact representation than a simple concatenation of the last contexts. Due to sensory uncertainty and partial observation, the state is also a random vector. The internal state of the input last context is represented as

$$
s(t)=f(l(t))
$$

where the function $f$ dynamically generates and updates states using: 1) temporal sliding window; 2) attention selection imple- 


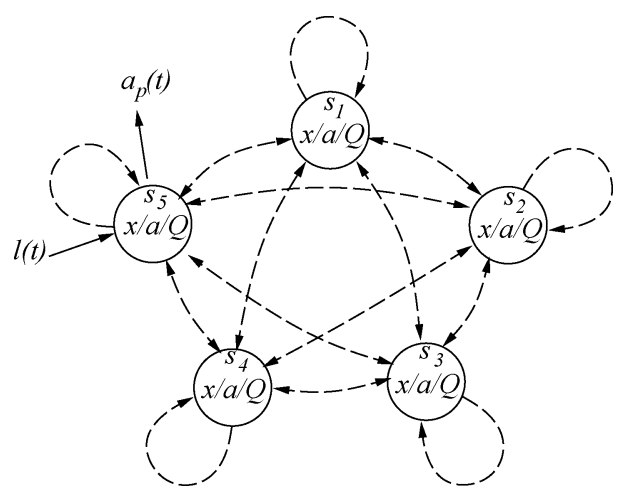

Fig. 7. Observation-driven Markov decision process with dynamically generated states, context-driven state representation, and value-based action generation. Each state corresponds to patterns of neural response. Note: the state is not symbolic. It is a vector in a high-dimensional space.

mented as internal action; 3 ) feature derivation (extraction); and 4) vector quantization. Functions 1) and 2) are implemented by the agent architecture to be presented. Functions 3) and 4) are implemented by IHDR which is part of the architecture.

Given any input last context $l$, the IHDR tree is used to search for the "best matched" microcluster $s$ in a leaf node of the tree, as the state of sensorimotor system. The matched vector $s$ is associated with a list of primed contexts

$$
s \rightarrow\left\{p_{1}, p_{2}, \ldots, p_{k}\right\} .
$$

This is how the regressor $R$ in (5) was implemented by IHDR.

Our agent model SAIL has multiple levels. Each level, with IHDR as its central mapping engine, realizes a mapping from the state space $S$ to the power set of the primed space $P=$ $\left\{\left(x^{\prime}, a^{\prime}, Q\right) \mid x^{\prime} \in X, a^{\prime} \in A, Q \in R\right\}$, where $x^{\prime}, a^{\prime}$, and $Q$ are a primed sensation, primed action, and a primed value, respectively, and $X, A$, and $R$ are their spaces, respectively. In other words, each level maps each prototype state to a list of primed sensation-action-value tuples. Collectively, $p=\left(x^{\prime}, a^{\prime}, Q\right)$ is called a primed context. The primed sensation (mental image) is useful for developing a high-level value system and for planning. The primed action is a possible action at the current state, and $Q$ is its value, estimated by the value system.

The overt behavior of the agent is mediated by a value system. To evaluate the value of each action $a$ at each state $s$, a $Q$-value function $Q(s, a)$ realizes a mapping $q: S \times A \rightarrow Q$, where $A$ is the action space and $Q$ is a scalar representing the estimated value. The innate value system is such that at the current state $s$, the agent executes the action $a$ that has the highest estimated $Q(s, a)$ value: $a^{*}=\max _{a \in A}\{Q(s, a)\}$.

The above way of modeling a decision process, as shown in Fig. 7, is called the observation-driven Markov model (ODMM) [32], [33]. Different from the traditional MDPs and HMMs, all the states here are numerical instead of symbolic. They are generated and updated "on-the-fly" from experience. Therefore, it is called developmental observation-driven MDP. Further, the state here represents the agent's internal context, instead of the state of a world event as in all existing nondevelopmental agents. However, like Q-learning, the SAIL robot's developmental program does not estimate the state transition probability, since otherwise the algorithm is not scalable to large memory: the

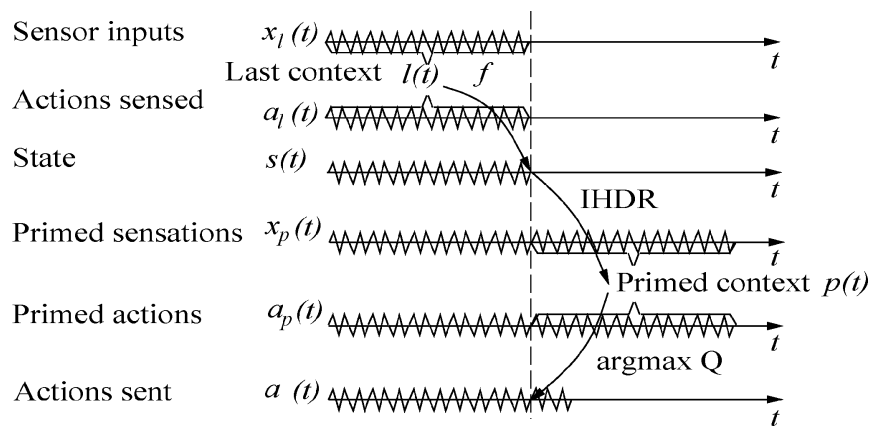

Fig. 8. A temporal illustration of the agent model. The last context includes information from sensors and the status of the agent's own actions. Depending on both the last context and the last state, the IHDR finds a new state associated with a list of primed contexts. The primed context with the highest primed value is selected and the corresponding primed action part is sent to the motors.

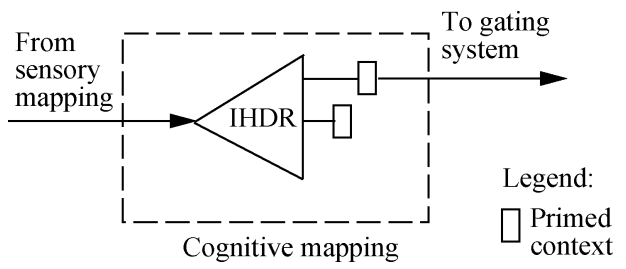

Fig. 9. The cognitive mapping module.

number of probability transitions of $n$ states is in the order of $O\left(n^{2}\right)$.

Using IHDR, the robot does the following in each execution cycle at time instance $t$.

1) Collects the last sensation $x_{l}(t)$ and the last action $a_{l}(t)$.

2) Constructs the last context $l(t)=\left(x_{l}(t), a_{l}(t)\right)$ as input to the IHDR tree.

3) The IHDR tree finds the best matched microcluster of $l(t)$ and denotes it as $s(t)$, which is the current state.

4) Gets the list of primed contexts in $P$, associated with $s(t)$.

5) Chooses the primed contexts $p(t)$ with the highest associated value.

Fig. 8 gives an illustration of this process. The module doing this job is called a cognitive mapping module (Fig. 9).

With this agent model, we have demonstrated that a robot can learn the association between a complex percept (such as vision and audition) and the control signals in real-time [34], [35]. In these processes, the environment is sensed by a robot as continuous and unsegmented data streams (e.g., the audio stream from a microphone, visual streams from cameras, and tactile streams from touch sensors). The internal self-organization facilitated by the architecture enables experience-based generalization.

\section{B. Handling the Missing Context}

The architecture module shown in Fig. 9 is essentially an online adaptive associator from each context input. It cannot look ahead and predict sufficiently far. If a follow-up context is missing, the IHDR tree will give a badly matched state and the agent's behavior is not predictable. To handle the missing context, we augmented the cognitive module by adding another IHDR tree (Fig. 10). The new tree is identical to the old one in the architecture except that it is associated with a prototype updating quеие (PUQ). We call the original tree the reality tree 


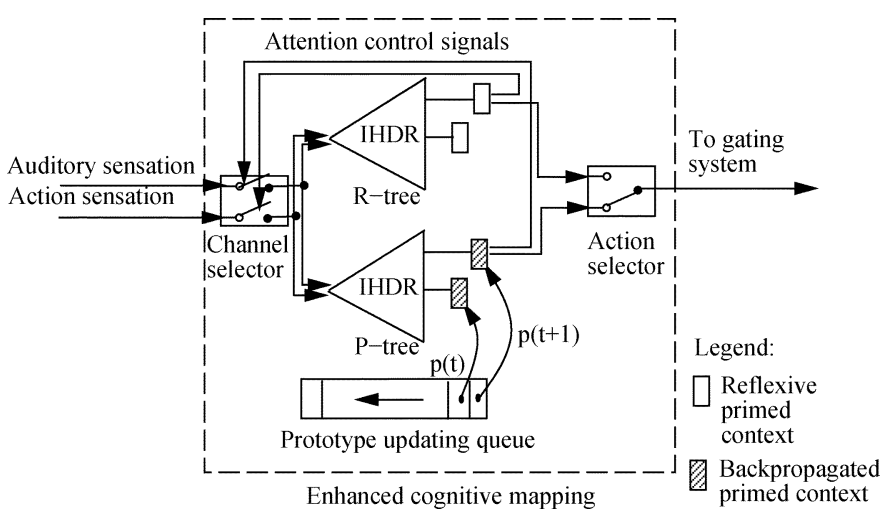

Fig. 10. The enhanced cognitive mapping module.

or $R$-tree and the added one the priming tree or P-tree. The goal of PUQ for the P-tree is to enable a looking-ahead (farther priming) mechanism. The PUQ maintains a list of pointers to the sequence of consecutive primed context retrieved by the P-tree. At every time instance, a pointer to a newly retrieved primed context enters the PUQ from the right end, while the oldest one moves out from the left end. When the pointers are kept in PUQ, the primed contexts they point to are updated with a recursive model adapted from Q-learning [30]

$$
p^{(n)}(t)=p^{(n-1)}(t)+\frac{1+w}{n}\left(\gamma p^{(n-1)}(t+1)-p^{(n-1)}(t)\right)
$$

where $p^{(n)}(t)$ is the primed context at time instance $t, n$ represents the number of times $p^{(n)}(t)$ has been updated, and $\gamma$ is a time-discount rate $(0<\gamma<1) . w$ is an amnesic parameter used to give more weight on the newer data points, which is typically positive, e.g., $w=2$.

Reorganizing (13), we have

$$
p^{(n)}(t)=\frac{n-1-w}{n} p^{(n-1)}(t)+\frac{1+w}{n} \gamma p^{(n-1)}(t+1)
$$

which shows more clearly that a primed context $p^{(n)}(t)$ is updated by averaging its last version $p^{(n-1)}(t)$ and the time-discounted version of the current primed context $p^{(n-1)}(t+1)$. In this way, the information embedded in the future context, $p^{(n-1)}(t+1)$ in model (13), is recursively backpropagated into earlier primed contexts. Therefore, (13) is effectively a prediction model. When an earlier context is recalled, it contains the expected future information. Note that this kind of manipulation on context can only be done on a numerical representation, which makes the power of numerical representation clear.

To view this effect more intuitively, we show the behavior of the prediction model in a simple example. Suppose the primed contexts appearing over time are represented by a series of scalars. A scalar with a value of 1 means the primed context contains certain information, while 0 means no information is embedded. An example of a series of the primed contexts is shown with a solid line in Fig. 11(a), where there is certain information over the five consecutive time instances $(t=55,56, \ldots, 59)$ and nothing elsewhere. Applying the model (13) with $\gamma=0.9, w=0$, and a PUQ of size 30 , we get the dotted line in Fig. 11(a) where the information has been backpropagated with the peak appearing at $t=54$. In other

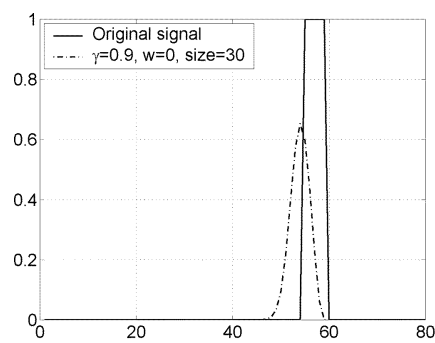

(a)

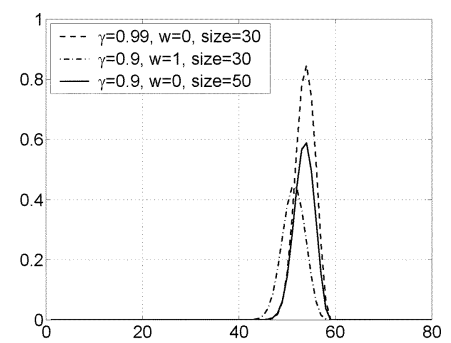

(b)
Fig. 11. The behavior of prediction model (13) with different $\gamma, w$, and PUQ size.

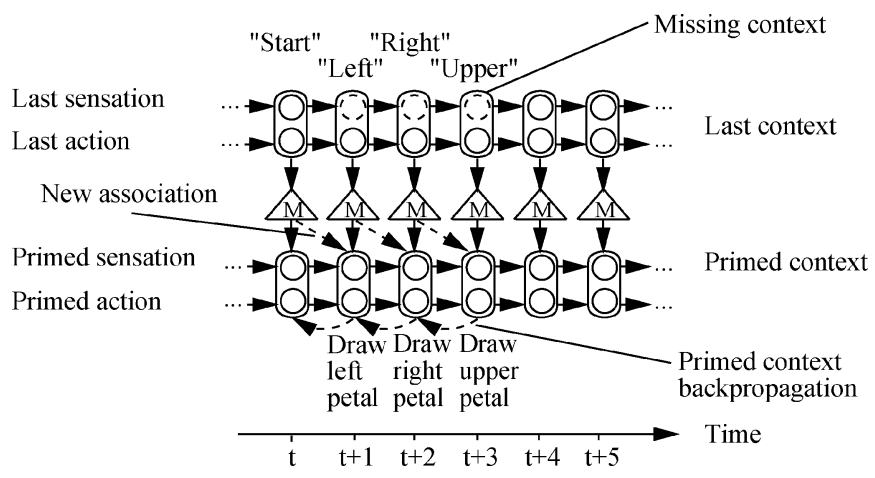

Fig. 12. Handling the missing context.

words, at an earlier time instance, the model predicts about $65 \%$ of the information.

Three parameters have influence on the behavior of model (13): the time-discount rate $\gamma$, the amnesic parameter $w$, and the size of the PUQ. As shown in Fig. 11(b), a larger $\gamma$ discounts future information, while a larger $w$ enables the model to use higher weight for the newly propagated data (faster learning). The performance is not very sensitive to the PUQ size, but the PUQ should be long enough so that the newly entered primed context can still affect the one that is about be moved out of the PUQ.

With the prediction model (13), the process of handling missing context can be viewed in Fig. 12. At each time instance, the robot decides the primed context, especially the primed action, based upon the observed last context. At the same time, the operations in PUQ enable the primed contexts to be backpropagated over time. When there is a missing context, e.g., missing "Left," "Right," and "Upper," the primed action will still be executed because of the newly established association between the last contexts and the primed contexts in the P-tree, shown in Fig. 12 as "new association." Therefore, one way to look at the roles played by the R-tree and the P-tree is as follows: both trees do the mapping from the last context to the primed context. While the R-tree primes the immediate mapping, the P-tree primes the near future.

\section{The Value System}

One problem of having a double-tree system in the cognitive mapping module is decision making. When both trees provide action vectors, which action should the agent choose and send to the effectors? This is the responsibility of the value system. 
Legend:
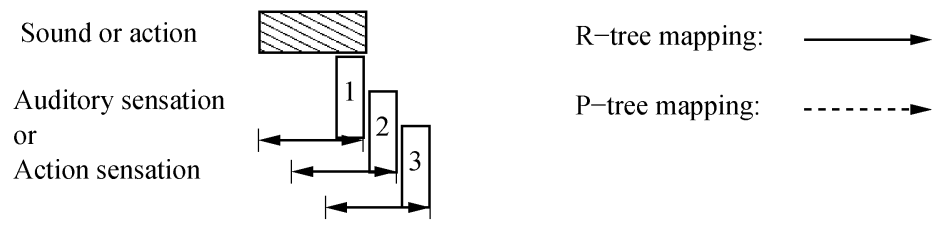

Without Attention:

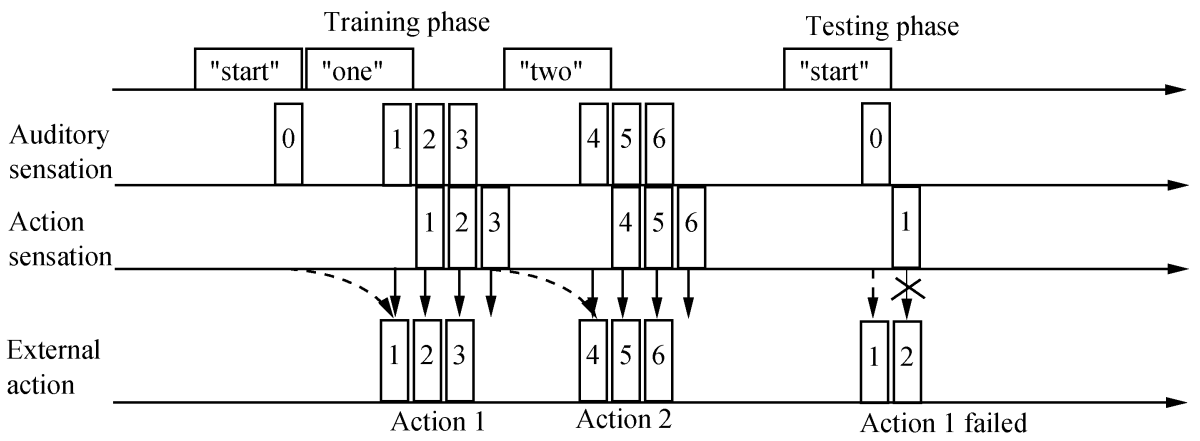

With Attention:

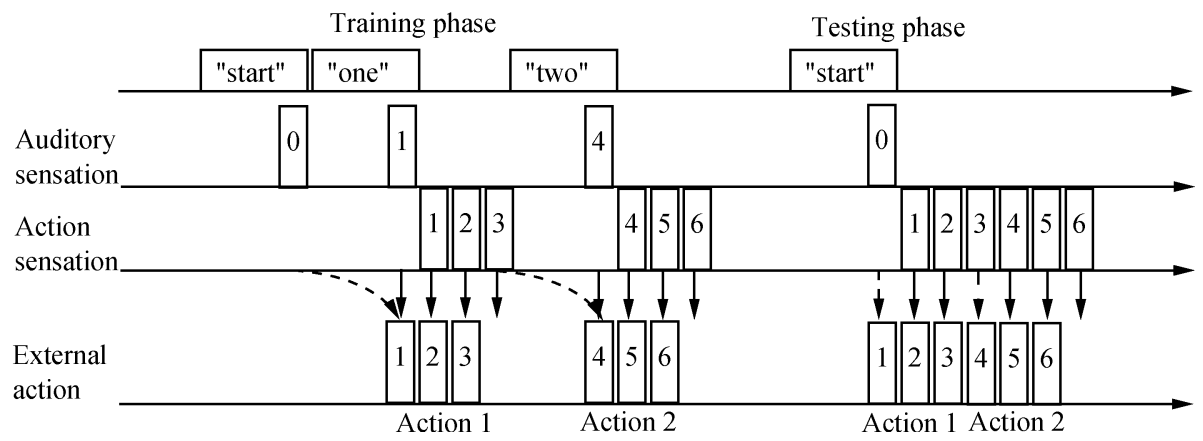

Fig. 13. The comparison of a system with and without intermodal attention.

Subsumption [36] is a simple example of a value system, while the state-action value in Q-learning is another one.

In the work presented, each primed context $p$ is associated with a primed value $Q(p)$. An initial value is assigned when a prototype is stored in the IHDR tree. If there is an action imposed at the time, the initial value is set to be 1 . Otherwise, it is 0 . Since the prediction model (13) is also applied to this value when the primed context enters the PUQ, the value will be distributed among consecutive primed contexts. In addition to this value, another factor affecting decision making is the degree of the match $d(s)$, the Euclidean distance between the last context $l$, and the prototype state $s$ retrieved by the tree. A small $d(s)$ means that the agent recalls the new context with a high confidence and, therefore, the decision is reliable.

Put the above two items together, we build a quantity called confidence index

$$
i(t)=\frac{Q(p(t))}{d(s(t))+\varepsilon}
$$

where $\varepsilon$ is a small number to avoid a zero denominator. The action selector in Fig. 10 compares the confidence indices associated with the action vectors given by both the R-tree and the P-tree. If the higher confidence index is larger than a preset threshold, the corresponding action vector gets through and goes to the corresponding effector.

\section{Intermodal Attention}

We have two trees doing different mappings and a value system selecting the outputs from them. The robot is still unable to do task transfer without a intermodal attention mechanism, as shown in Fig. 2.

In the work presented here, we consider two major sensory modalities. The external one is audition, and the internal one is the sensation of the agent action. The internal sensation is important because it informs the agent its current status of action which may affect its later behavior, as discussed in Section II-A. One way of treating these two modalities is to put the sensation vectors together as a single last context entering the IHDR tree. However, we will see that such a naive method does not work.

First, we need to understand that a last context covers a short duration instead of a single time instance. That means there is still some nonsilent auditory sensation presented to the robot even when an utterance is finished. The same thing happens to the action sensation. To graphically explain this, we represent the (auditory or action) sensation with a series of numbered bars in Fig. 13. As shown in the legend of Fig. 13, each numbered bar covers the sensation over a short history.

Since there are two sensing modalities, the last context at each time instance is described by a pair of numbered bars, each for the auditory sensation and the action sensation, respectively. For example, the first last context shown in Fig. 13 is $\langle$ bar 0 , blank $\rangle$, 
where bar 0 represents the last auditory sensation and blank represents the last action sensation. The external action is also presented as a numbered bar in Fig. 13. Notice that the sensed action is the external action at the last time instance. Therefore, the action sensation is a shifted bar sequence of the external action. In general, a blank bar means that no sound or action is sensed or executed.

During training, the teacher gives all three commands, "start" $\left(C_{c}\right)$, "one" $\left(C_{s 1}\right)$, and "two" $\left(C_{s 2}\right)$. The R-tree learns the immediate mapping from a last context to a primed context which includes a primed action. This mapping is shown by the solid arrows in Fig. 13. The P-tree learns a mapping with prediction to a near future, as shown with the dash arrows in Fig. 13. The choice to execute the primed action of the R-tree or the P-tree is made by the value system.

Now, we take a look at a system running without an intermodal attention. During testing, after the utterance of "start," the P-tree successfully gives the mapping $\langle$ bar 0, blank $\rangle \rightarrow\langle$ bar 1$\rangle$ and the value system chooses the output of the P-tree as the external action. Everything is perfect so far. Unfortunately, the last context configuration in the next time instance $\langle$ blank, bar 1$\rangle$ has never showed up during training, leading to unpredictable behavior. Then, the new task skill fails to be acquired.

A intermodal attention mechanism will resolve the problem. Notice that although $\langle$ blank, bar 1$\rangle$ has never appeared during training, the action bar 1 is always followed by the action bar 2. Suppose the robot pays attention to its action sensation only (the action bar 1) after it starts to move during training. Then, the auditory bar 2 and bar 3 will not appear in the auditory sensation part of the last context configuration. Now, we have the last context configuration of $\langle$ blank, bar 1$\rangle$ that is mapped to the external action $\langle b a r 2\rangle$ by the R-tree. When action 1 is finished, the attention to auditory sensation should be restored to sense the utterance of "two," and the similar attention switch to action sensation is needed after action 2 starts to be executed. In general, the selective attention brings related information to the recognition process.

In summary, we need an attention selection mechanism that can switch between the two sensation channels. A principle for attention selection is that the channel with higher short-term variation is more salient and would receive the exclusive attention. To do this, we designed a simple intermodal attention module, the channel selector shown in Fig. 10. The eligibility of each modality channel (candidate for attention) is defined as the standard deviation $\sigma(t)$ of the magnitude $m(t)$ of the sensation from this channel, computed incrementally as

$$
\sigma(t)=\frac{t-1-w}{t} \sigma(t-1)+\frac{1+w}{t} m(t)
$$

where $w$ is the amnesic parameter to give more weight to the new samples. With an appropriate $w, \sigma(t)$ would represent the short-term variation of the sensation. The channel selector implements a "winner-takes-all" criterion based on the eligibility. It passes the signals from the winning channel and blocks the signals from the losing channel by replacing it with zero vectors.

\section{E. Single-Level Algorithm}

As a summary of the above, at each time instance, the agent executes the following learning process repeatedly until the power is off. Since there are no explicitly separated training and testing sessions, the algorithm is executed while the agent is "alive."

1) Collect the sensation from both the auditory sensor $x_{l}(t)$, and the action sensor $a_{l}(t)$.

2) The channel attention selector (Fig. 10) forms a single sensation vector (the last context), $l(t)=\left(x_{l}(t), a_{l}(t)\right)$, by replacing $x_{l}(t)$ or $a_{l}(t)$ with a zero vector.

3) Both the R-tree and the P-tree search the best matched prototype from $l(t)$, using their IHDR tree, respectively.

4) The best-matched states, or the prototypes $s_{R}(t)$ and $s_{P}(t)$ are retrieved from the two trees together with their associated primed contexts $p_{R}(t)$ and $p_{P}(t)$, respectively.

5) If there is an imposed action $a_{i}(t)$, the primed action part of $p_{R}(t)$ is set to be $a_{i}(t)$ and its associated value $Q$ is set to be 1 . Otherwise, the primed action part of $p_{R}(t)$ is set to be a zero vector (the default nonaction) and its associated value is also set to be 0 .

6) Compute the confidence index using (15) for both trees and decide the external action $a_{e}(t)$. Its associated value is termed as $Q_{e}(t)$.

7) The primed context from the P-tree $p_{P}(t)$ enters the PUQ and each entry in the PUQ is updated from right to left according to model (13). Specifically, the sensation part is updated using $s_{P}(t)$, the action part using $a_{e}(t)$, and the value part using $Q_{e}(t)$, recursively through the PUQ.

8) Send $a_{e}(t)$ to the corresponding effectors.

\section{Multilevel ArChiteCture}

The artificial agent with the single-level architecture effectively learned simple actions and developed complex behaviors through communicative learning, as demonstrated in Section V. Since the PUQ backpropagates primed contexts which include the primed value, the single-level architecture, in theory, is capable of behavior shaping through the reinforcement feedback, which is important in order to allow teacher errors or to allow the learner to explore on its own. However, the preliminary experiments were not as successful as we expected.

A further investigation showed that the difficulties originated from the practice of the numeric representation. Due to the prediction model (13) and the PUQ, the primed context of a particular primitive prototype would be propagated to other prototypes so that multiple primitive prototypes may have a similar primed context. In the real-world application, exact contexts are not guaranteed to be repeated, which means all the propagated primitive prototypes may not show up in the following training session. As a result, while we may adjust the primed contexts of some primitive prototypes, we may not be able to adjust all the propagated ones. In other words, with the numeric representation, a new challenge is to generalize the sensorimotor associations without tedious prototype-by-prototype learning, which we call the "abstraction-from-signals" issue.

To solve this problem, we designed an architecture with multiple levels of sensorimotor systems, as shown in Fig. 14. The 


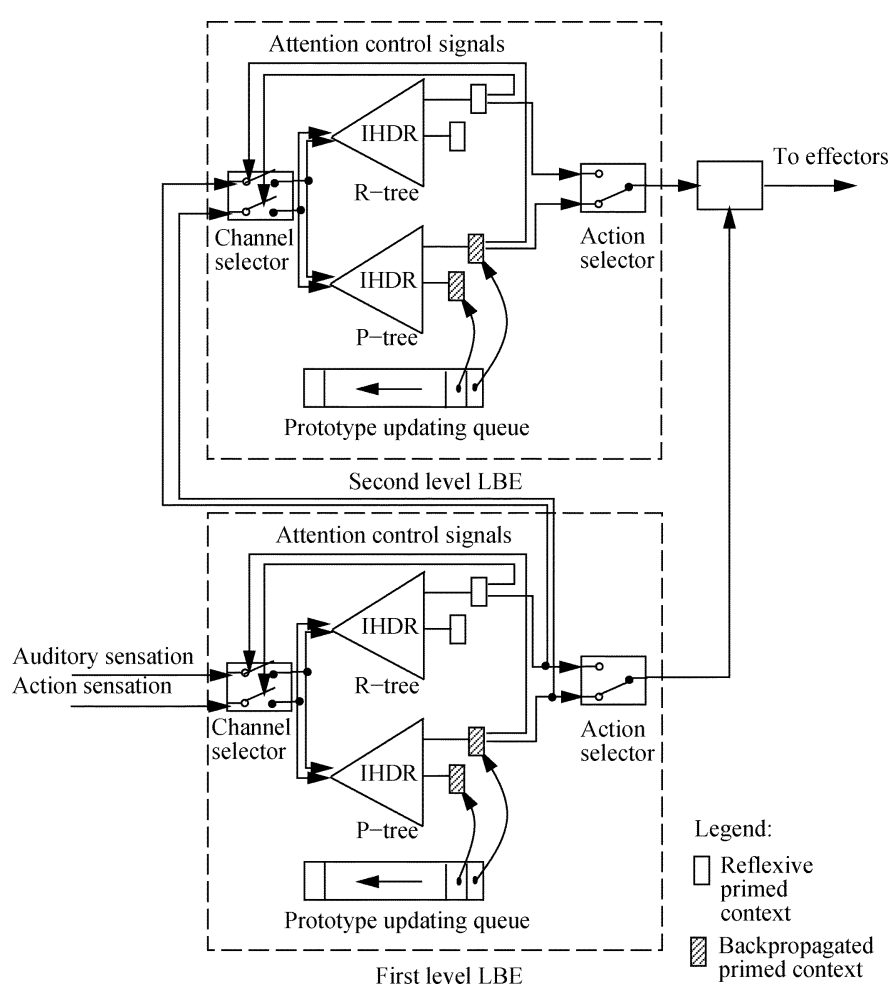

Fig. 14. A two-level architecture of SAIL.

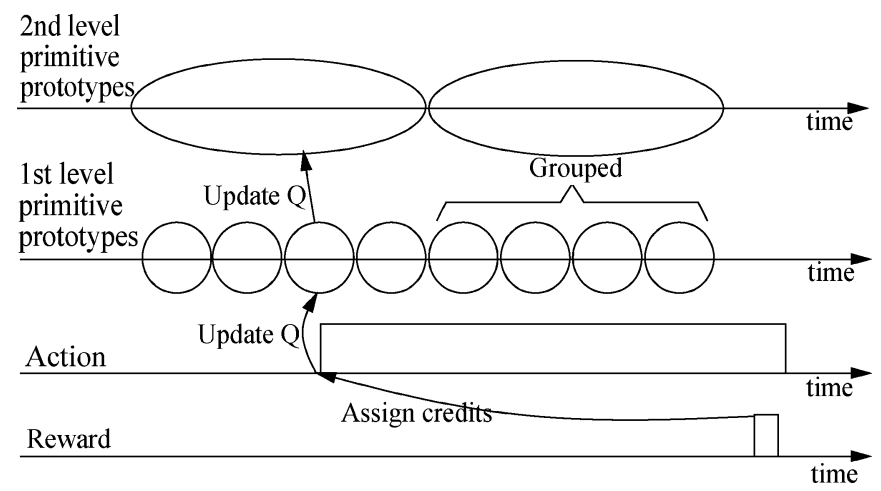

Fig. 15. Internal mechanism of the two-level architecture.

higher level one had coarse granularity both in space and in time. The function of the higher level system was to evaluate and tune the behaviors of the lower level system.

We call the whole module of Fig. 10 the level building element (LBE) corresponding to a single sensorimotor system. The new architecture has two levels of LBEs. All of the LBEs run the algorithm of Section III-E, except that the second-level one takes the primed sensations of the lower level one as the input. The underlying idea of the two-level system is as follows: Due to the prediction model (13), the primed sensation vectors are an averaged version of its future context vectors. This means that the vectors which used to fall into different primitive prototypes in the first level LBE $L_{1}$ may be grouped into one primitive prototype of the second level LBE $L_{2}$, as shown in Fig. 15. As a result, even though it may be rare for a particular primitive prototype to be revisited in $L_{1}$, it is very likely for $L_{2}$ to cover the context represented by this primitive prototype when a similar context appears. The above soft abstraction enables the generalization.

To choose actions from multiple levels, $L_{2}$ checks the $Q$ value of the action from $L_{2}$ that is closest to the one selected by $L_{1}$. If it is larger than zero, the corresponding control signals would be sent to the effectors. Otherwise, the action would be blocked. In other words, no action would be taken. The $Q$ value given by $L_{2}$ will enter $L_{1}$ as an internal reward so the behavior of $L_{1}$ will be tuned even if there is no constant environment feedback.

In the current implementation, the R-tree of $L_{2}$ was not used. It will be used when an even higher level is built through further development. To quickly assign the reward to the right context, a credit-assignment criterion is preprogrammed by targeting the reward to the starting point of a recently conducted action, as shown in Fig. 15.

\section{A. Multilevel Algorithm}

In summary, the following is the developmental learning algorithm of the two-level system at each time instance. The procedure is run repeatedly until the power is off.

1) Collect the sensation from both the auditory sensor $x_{l}(t)$, and the action sensor $a_{l}(t)$.

2) For $L_{1}$, do steps 2) through 5) of the single-level algorithm of Section III-E. Denote the primitive prototypes retrieved by R-tree and P-tree as $s_{R 1}(t)$ and $s_{P 1}(t)$, respectively. Find the primed context with highest confident index among the primed contexts associated with $s_{R 1}(t)$ and $s_{P 1}(t)$ and denote it as $p_{1}(t)$.

3) Take the primed sensation part of $p_{1}(t)$ as the input to $L_{2}$ and do steps 2) through 5) of the single-level algorithm of Section III-E for $L_{2}$. Denote the primitive prototype retrieved by P-tree as $s_{P 2}(t)$. Find the primed context of $s_{P 2}(t)$ with the primed action part most similar to that of $p_{1}(t)$ and denote it as $p_{2}(t)$. Let $p_{2}(t)$ enter the PUQ of $L_{2}$ and update according to model (13). For updating $Q$, use

$Q^{(n)}(t)=Q^{(n-1)}(t)+\frac{1+l}{n}\left[r(t)+\gamma Q^{(n-1)}(t+1)-Q^{(n-1)}(t)\right]$.

4) If the $Q$ value of $p_{2}(t)$ is larger than zero, send the primed action part of $p_{1}(t)$ to the corresponding effectors. Otherwise, send the zero vector to the corresponding effectors.

5) Let $p_{1}(t)$ enter the PUQ of $L_{1}$ and update according to model (13). For updating $Q$, use

$$
\begin{aligned}
& Q^{(n)}(t)=Q^{(n-1)}(t) \\
& \quad+\frac{1+w}{n}\left[r(t)+Q_{2}^{(n-1)}(t)+\gamma Q^{(n-1)}(t+1)-Q^{(n-1)}(t)\right]
\end{aligned}
$$

where $Q_{2}^{(n)}(t)$ is the $Q$ value of $p_{2}(t)$.

From the above discussions, we can see that the role of $L_{2}$ is effectively a module evaluating the behaviors of $L_{1}$. This means that our system has some characteristics of the wellknown actor-critic methods [37, p. 151]. The major differences here are: 1) our method starts from raw sensors instead of symbolic input; 2) both the "critic" $L_{2}$ and the "actor" $L_{1}$ learn from the environment; and 3) the critic is not task-specific. It gives the system a quicker response to the change of the environment by not allowing the actor to wait until the critic realizes the change. Another important characteristic of our system 


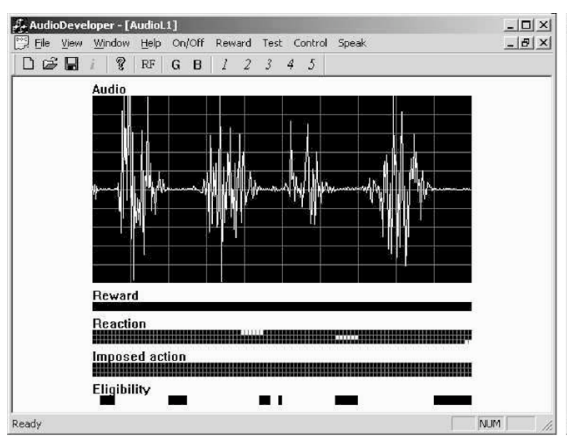

(a)

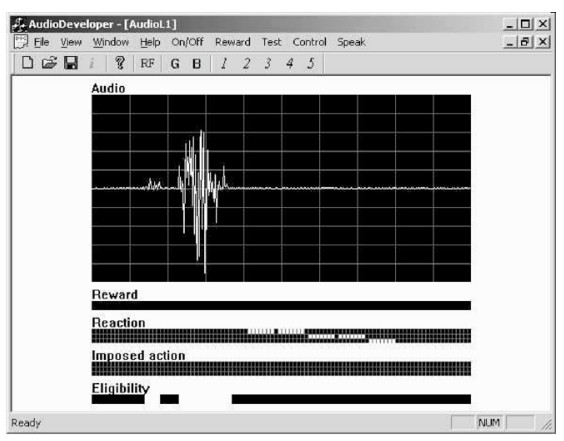

(b)

Fig. 16. The GUI of AudioDeveloper. (a) During online learning. (b) After online learning.

is that the "critic" and the "actor" have similar architecture and learn in the same manner, which makes the architecture more systematic. $L_{2}$ was motivated by higher order cortex in the biological system [38], but further discussion is beyond the scope of this paper.

\section{EXPERIMENTS ON THE AUDIODEVELOPER SOFTWARE AGENT}

The AudioDeveloper is a real-time (software) agent that implements the proposed agent model to learn basic sensorimotor skills and do task transfer. Everything outside the agent is called environment, which includes the physical world around the PC on which AudioDeveloper is running. The human teacher is part of the environment.

The agent can interact with the environment only through its sensors and effectors, and there exist no other channels between the agent and the environment. The sensors include the following.

1) An external microphone. It is called an (innately) unbiased sensor since the value system does not associate its input to any particular value at the birth time.

2) An external reward sensor. It is an (innately) biased sensor because the value system associates its input to particular values: 1 is good and -1 is bad. At each state $s$, the value system selects action $a$ that leads to highest estimated value $Q(s, a)$. It resembles a combination of "sweet sensor" and "pain sensor."

3) An internal sensor for every external effector to sense the control value of that effector. In the sensorimotor system, such sensors are realized by neural projections, and not realized by neural receptors. However, for clarification, the SASE agent [26], [25] must sense its own actions so that its decision can depend on the status of the external effectors.

4) An internal sensor for every internal effector. In the work presented here, the only internal effector is the intermodel attention selection, i.e., paying attention to the microphone input (external) or to its own action (internal).

The effectors include the following.

1) Three external action effectors, named $e_{1}, e_{2}, e_{3}$, etc., simulating a three-joint arm. Each takes the value 0 or 1 at any time. When $e_{i}=1$, the joint action represented by $e_{i}$ is "carried on," $i=1,2,3$. Otherwise, the action is not carried on.
2) A single internal effector, the intermodel attention selector as described above.

The sound was digitized at $11.025 \mathrm{kHz}$. The 13-order Melfrequency Cepstral Coefficients (MFCCs) [39] were computed over every auditory frame of 256 sample points. Two consecutive auditory frames overlapped each other with 56 sample points. Therefore, the start points of each two consecutive auditory frame is separated by 200 sample points (about $18.1 \mathrm{~ms}$ ), which was also the interval for each execution cycle in the enhanced cognitive mapping. In order to cover a short speech phrase, each last context composed of 30 consecutive auditory frames (about $0.54 \mathrm{~s}$ ), which amounts to an auditory feature vector of $30 \times 13=390$ dimensions. The reward sensor, the external effector sensor, and the internal effector sensor have the dimensions of one, three, and one, respectively, within each auditory frame. For every vector of last context, the total dimension is $390+30 \times(1+3+1)=540$.

We need to simulate a compliant arm where its action can be imposed by a teacher. For example, the mother can hold the arm of her child to teach him how to draw. Through the AudioDeveloper, we allow the human teacher to directly assign value to every external effector in real-time through the GUI, which is directly executed by the corresponding external effector and sensed by the dedicated internal sensor. However, such external imposition of action cannot be applied to internal effectors. For example, the mother cannot impose a control value to the internal attention selector of her child. In other words, action for internal effectors must either be innate reflex (programmed) or learned without external imposition (e.g., through reinforcement learning). In our work, the internal effector, attention selection, has an innate reflex based on the competition of two channels: The channel with the larger amount of recent relative change wins the attention.

The simulated agent AudioDeveloper provides a detailed performance record and ground truth that are not readily available from a real robot. The AudioDeveloper served as a testbed for the developmental program before the implementation of the real robot.

In the GUI (Fig. 16), the top panel shows the audio waves from the microphone. The rewards, reactions (action of the agent), the imposed actions (from the teacher), and the eligibility (information used by internal attention selection) are shown in real-time in the second to fifth panel. The horizontal axis is the real-time. 
The developmental program was designed and implemented without any task-specific information. The agent learns while interacting with the real world. The purpose of each training session is in the mind of the trainer only. The trainer creates arranged experience to enable the AudioDeveloper to acquire the desired sensorimotor skills and to use those skills to continuously develop more complex skills by transferring previously learned skills.

In next section, we first present an experiment that involves a single speaker. In Section V-B, we present an experiment that involves multiple speakers to demonstrate the scalability of the proposed method to multiple speakers without a need to rewrite the developmental program.

\section{A. Single-Speaker Experiment}

The single-speaker experiment is divided into two steps. (1) The agent learns basic sensorimotor skills by following verbal commands. (2) The agent learns complex sensorimotor skills by following new verbal commands through task transfer. All of the learning of the above two steps is done in the same operational mode as required by development, i.e., real-time physical interactions between the agent and the environment. As discussed above, whether an interaction is for training or testing is only in the mind of the teacher. In the following discussion, when we mention the test session, we simply mean "freezing" any changes to the cognitive mapping module so that we may evaluate the developed system.

The procedure of the training session of Step 1) is as follows.

1) A verbal command is spoken to the AudioDeveloper.

2) At the end of the utterance, a corresponding action is imposed by pressing a button in the toolbar of the AudioDeveloper.

3) Wait for 3-4 s and go back to $1 .^{2}$

The above training process is a grounded speech learning step that has been discussed in detail in our developmental audition work [21], [35]. This is challenging because the verbal utterance has a certain temporal duration (about $0.5 \mathrm{~s}$ ) and the imposed action is delayed randomly within a small time window. Each utterance produces more than 50 vectors as contexts $l$, including partial covering of the utterance during the onset and offset of the utterance. These 50 or so context vectors will produce an unknown number of prototype vectors $s$ in the long-term memory of IHDR. Reliable generalization from these unknown numbers of prototypes to the correct motor actions in real-time online settings is very challenging [21], [35].

While supervised learning (i.e., imposed actions in real-time) was conducted in this experiment, the trainer can also use a reinforcer, as we did in [40], but this requires exploration of actions so that the value of every action can be estimated. Therefore, training using exploration and a reinforcer is expensive.

After training, the verbal commands were spoken to the $\mathrm{Au}$ dioDeveloper again with no actions imposed. As shown in the

\footnotetext{
${ }^{2}$ This separation period is to make sure the two consecutive commands and actions are well separated from each other. As long as this separation requirement is fulfilled, the exact length of the period will not impact the performance of agent. This principle also applies to the training sessions we will introduce in the following sections.
}

experimental results below, the AudioDeveloper correctly conducted the appropriate action one, action two, and action three, after the verbal commands, "one," "two," and "three," respectively. This completes Step 1), learning basic skills.

The training session of Step 2), learning more complex skills from transfer of previously learned sensorimotor skills, is as follows. While continuously running the agent which has learned the basic sensorimotor skills, the trainer does the following.

1) Speak verbal command "start" followed by verbal command "one," to the AudioDeveloper. The verbal command "one" will invoke action one based on previously acquired sensorimotor skills. Perform a few practices. Wait for 3-4 s between each practice.

2) Speak verbal command "start" only. If the agent carried out action one, it is a success of transfer, based on the arranged experience. If action one does not follow the verbal command "start," repeat the above steps a few times more.

3) To transfer other skills into those complex sensorimotor skills, the trainer may speak verbal command "two" at the end of the action one which is now invoked by "start." The verbal command "two" will invoke action two based on the previously acquired sensorimotor skills. Perform a few practices. Wait for 3-4 s between each practice.

4) At the end of action one, do not speak verbal command "two." If the agent invokes action two at the end of action one, it is a success of transfer based on the arranged experience. Practice a few times.

5) The above can be repeated to extend the length of the desired action sequence, until the agent reliably responds to the verbal command "start" with the intended action sequence one, two, and three, without the need of verbal hints "one," "two," and "three." Practice a few times.

As can be seen, the trainer can speak the verbal hint for the next action as soon as the previous action ends. He can either extend the sequence one verbal hint at a time or train the entire sequence by providing verbal hints at the right contexts.

Fig. 17 shows a fraction of this training session. In Fig. 17, the upper panel presents the energy of the verbal commands along the time axis. The next three panels display the 3-D action vectors of the R-tree, the P-tree, and the enhanced cognitive mapping. The behaviors of the robot were identified by the component of the action vector with the maximum value. For example, if the first component of the action vector had the maximum value, it was identified as action 1 . The fifth panel of GUI shows the confidence of the R-tree and the P-tree on their outputs. The bottom panel shows the attention of the system over the auditory and action sensation channels. The sequence of the external action vectors shows that the system successfully responded to commands "one," "two," and "three," accordingly.

Let us take a closer look at how the system behaved around time instance 100 when the command "one" was being spoken (Fig. 18). Note that the confidence subplot is shown in a semilogarithmic way in this figure. At time instance 98, the P-tree started to show a significant trend to conduct action one and it had a higher confidence than the R-tree. So, the enhanced cognitive mapping fired the external action one. Once the action was started, the attention was switched from the auditory sensation channel to the action sensation channel. With the sensation for 

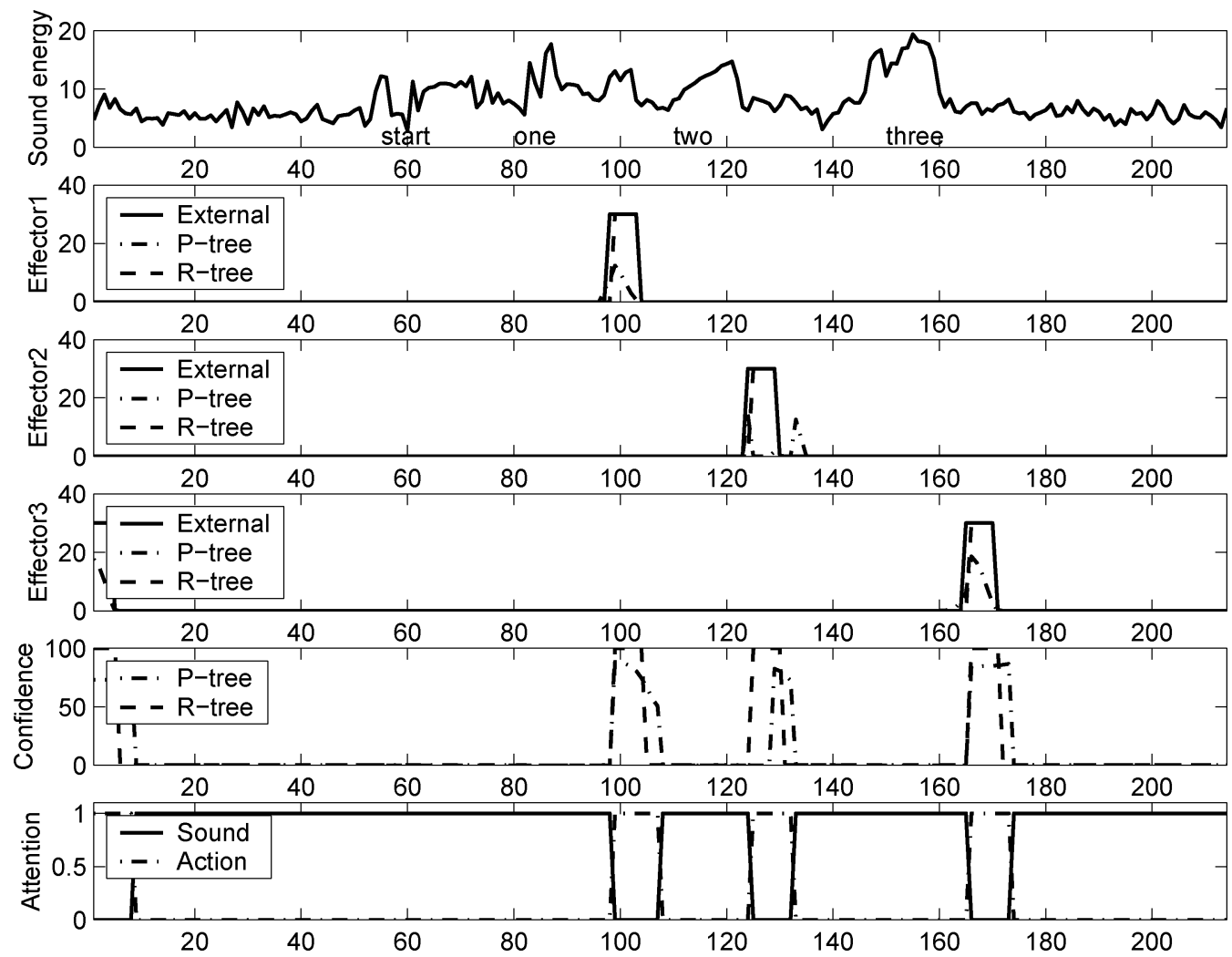

Fig. 17. A fraction of the training session of Step 2).

the starting of action one, the R-tree began to have higher confidence and continued to conduct action one until time instance 104 when the R-tree decided to stop the action. For a better view of the transition of the confidence of the R-tree and the P-tree between time instances 100 and 104, the reader is referred to Fig. 17.

After Step 2) training, the system has acquired more complex sonsorimotor skills. If only the composite command "start" was given, the system successfully repeated the action sequence, one, two, and three, as shown in Fig. 19. Again, let us give the system behavior a closer look as shown in Fig. 20, which expands the time axis around time instance $460 .{ }^{3}$ During the training, an utterance of "start" was always followed by an utterance of "one." Due to the backpropagation of the primed context, upon hearing "start," the P-tree learned to produce the primed context associated with "one." Therefore, without hearing "one" during testing, the P-tree started to have a significant trend of executing action one after hearing "start" at time instance 464. Since the P-tree also had a higher confidence than the R-tree at that time, action one was fired as the external action of the enhanced cognitive mapping. The attention was then switched to the action sensation channel at time instance 465 and the R-tree took over until action one was stopped at time instance 471, since it had a higher confidence (see Fig. 19 for an expanded view of confidence).

With the numerical representation, an utterance of a verbal command typically corresponds to multiple primitive prototypes saved in the IHDR tree, depending on the duration and the

\footnotetext{
${ }^{3}$ The confidence subplot is shown as semi-logarithmic.
}

auditory changes of the utterance. During training, the primed context of all of these multiple prototypes may be changed because of the primed context backpropagation. Therefore, there were multiple primitive prototypes in the P-tree to start action one. If the basic action was too short, it could be fired for a second time. That is why we see the action repeated after the system finished it once at time instance 472. In the real world, the duration of an action is typically longer than an utterance. So such action repetition would be avoided as shown on a real robot in the experiment below.

Similar to the case that "start" elicited action one during training, the P-tree "saw" that action one was followed by the utterance of "two" repeatedly. Therefore, the primed context of the verbal "two" was backpropagated to the last context of seeing action one. As a result, the P-tree started to show a high confidence of conducting action two at time instance 480 , although verbal "two" was not presented. The above process was repeated until the end of action three, which means the agent successfully learned to conduct the entire new task.

\section{B. Multiple-Speaker Experiment}

The above experimental results were obtained with the interaction between the AudioDeveloper and one speaker. To test the AudioDeveloper with wider sensory variance, we conducted an experiment involving multiple speakers. The procedure for training the agent is the same as the single-speaker experiment. The difference is how many speakers contributed to the auditory commands. 


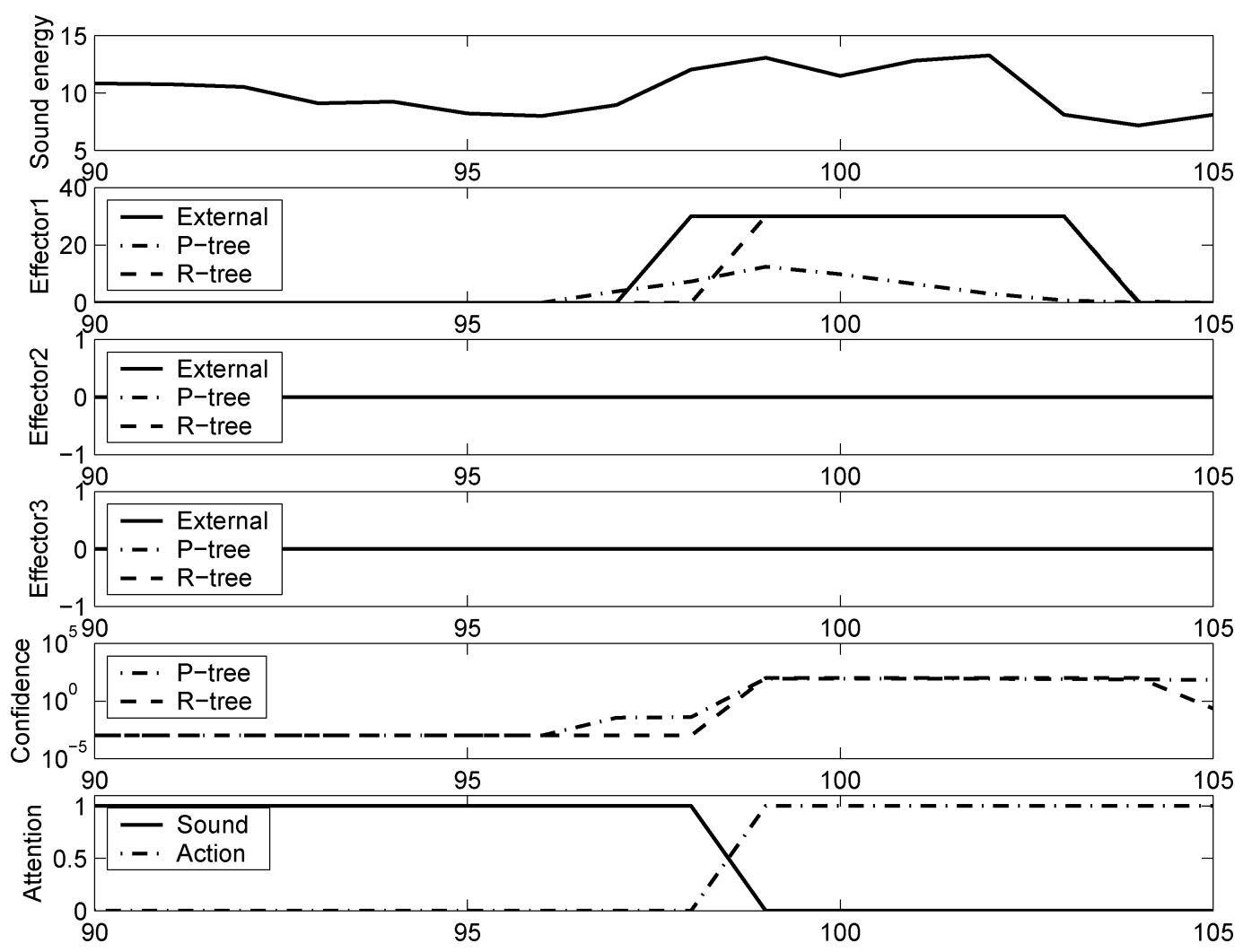

Fig. 18. A closer look at the training session of Step 2).

To save human efforts in training the AudioDeveloper, first we built another software agent, the AudioTeacher, as a "teacher." It played prerecorded sounds through the computer speaker and gave imposed actions to the AudioDeveloper through a messaging mechanism between processes. The auditory data was taken from the number data set contributed by 63 people with a variety of nationalities (American native English speakers and non-native English speakers who were Chinese, French, Indian, Malaysian, and Spanish) and ages (from 18 to 50). Each person made five utterances for each of the ten isolated numbers, one to ten. In this experiment, we only used the utterances of "one," "two," and "ten," where the first two were the simple commands and the last one was the composite command. Among the five utterances of each number, three were used in training and two were used in testing. In effect, in Step 2) testing, only the utterances of the composite command were needed.

The AudioTeacher was programmed to lead the two-step training process, as discussed in Section V-A. Since we did not have enough speech data from each subject, the data was replayed after each round. We call one cycle through the data set "an epoch." In Step 1) training, one epoch was sufficient for the AudioDeveloper to acquire the simple actions and reached a high correct rate $(>95 \%)$. The failed cases were those where the AudioDeveloper did not recognize the simple command. In these cases, the AudioDeveloper simply did not do anything since the action selector in Fig. 10 did not allow any action vectors to be passed to the effectors when the confidence index, which was a function of both the primed value and the goodness of match between a last context and a primitive prototype, was lower than a predefined threshold. In Step 2) training, the AudioDeveloper needed multiple epochs (reviews and practices) for the new task because of the need of backpropagation through the PUQ, similar to the need to establish a reliable temporal pairing as in child learning. We stopped the Step 2) training after 20 epochs. Starting from epoch six of the Step 2) training, the cognitive mapping was saved after each epoch for later performance evaluation.

The training process took about 6 hours for the 63 subjects' data. While the total storage size of the cognitive mapping modules reached $482 \mathrm{MB}$, the AudioDeveloper still worked in realtime thanks to the logarithmic time complexity of the IHDR trees. Specifically, the average execution time in each execution step is $4.6 \mathrm{~ms}$, well under the real-time interval of one frame of speech data, $18.1 \mathrm{~ms}$.

Although Step 1) training enabled the AudioDeveloper to conduct simple actions upon hearing simple commands, the composite command would not elicit any action of the AudioDeveloper in the beginning of Step 2) training. As the Step 2) training session went on, the composite command started to elicit the first simple action one, the first part of the new task. Denoted as C.R.1, the correct rate was evaluated as follows. If the AudioDeveloper made action one, once or several times after a composite command was spoken, we counted it as a successful acquisition of the first part of the new task. Otherwise, it was a failure. With more Step 2) training, C.R.1 gradually increased. In addition, the composite command started to elicit the whole new task, which was action one followed by action two. Denoted as C.R.2, this correct rate was evaluated as follows. After a composite command was spoken, 


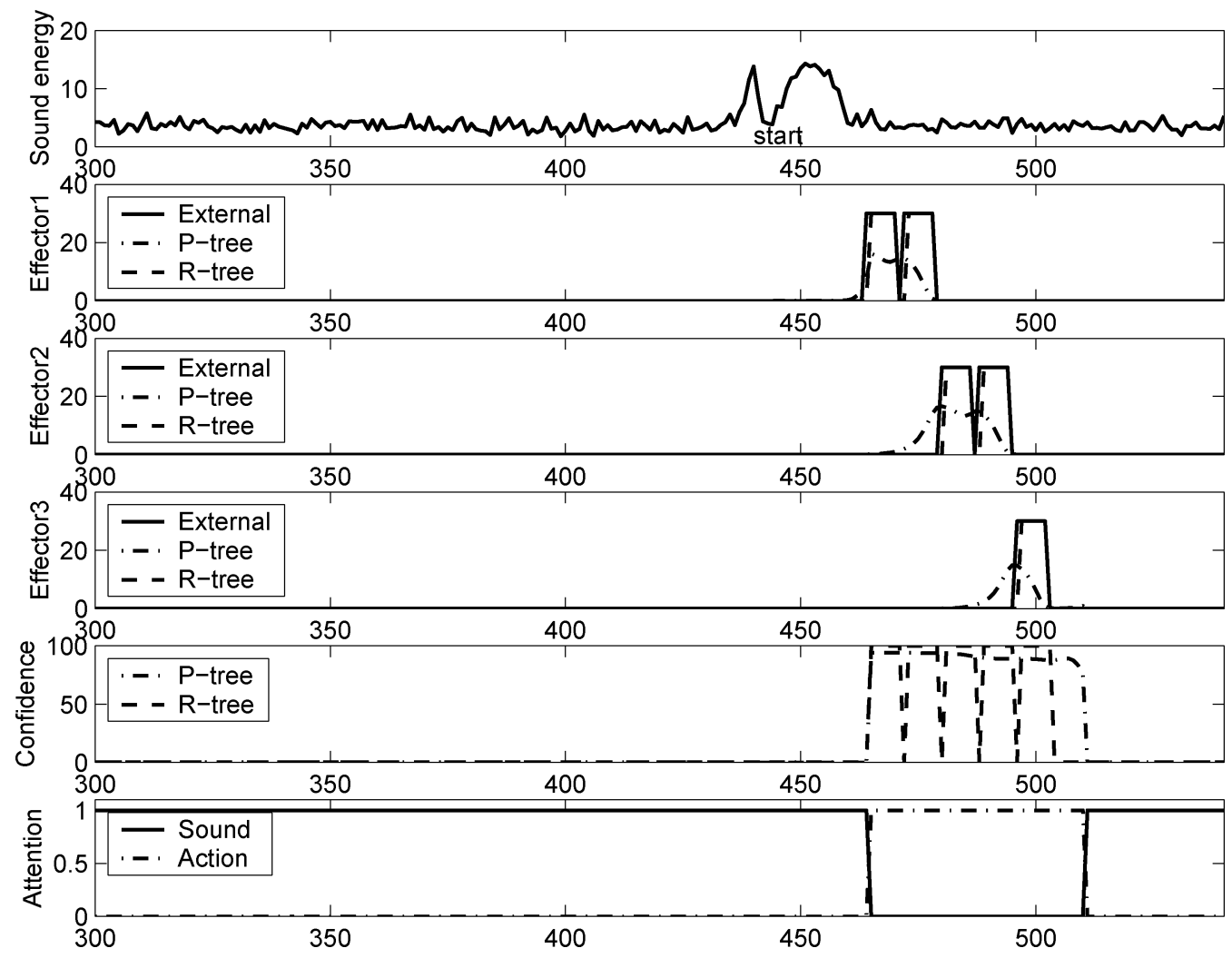

Fig. 19. A fraction of the test session of Step 2).

if the AudioDeveloper made action one, once or several times followed by action two, once or several times, we counted it as a successful acquisition of the new task. Otherwise, it was a failure. Table I shows the C.R.1 and C.R.2. after each epoch in the Step 2) training. As expected, action one was acquired earlier than the acquisition of the whole new task. After 20 epochs of Step 2) training, the AudioDeveloper learned the new task reliably with a correct response rate of about $93 \%$.

Note that the sensorimotor association for the composite command was realized by the P-tree, which involved the PUQ mechanism. As discussed in Section III-B, the function of PUQ was to backpropagate the primed context so that a last context could elicit a primed action far in the future. The backpropagation took time, or more precisely, required the reappearance of the last contexts of interest in order to update their primed contexts. Consequently, while the correct rate for the simple commands reached a high level very quickly ( $>95 \%$ after one epoch) because of the "one-instance" learning capability of IHDR, the correct rate for the composite command improved gradually to $93 \%$.

In the $7 \%$ of failed cases, the complex command did not elicit any actions of the AudioDeveloper. These 7\% of failed cases were related to three subjects. We examined them one by one and found that the reasons for failure were the same: these three subjects' utterances were very short. In the Step 2) training, the AudioTeacher was programmed to play the composite command and the simple commands one after the other. When the utterances were very short, the two consecutive commands sounded like one word to the AudioDeveloper. Apparently, the AudioDeveloper could not recognize these "strange" words and decided not to respond. Since the simple commands were not recognized correctly, the Step 2) training was not truly effective. After adjusting the separation time between the utterances for these three subjects to be about $0.5 \mathrm{~s}$, the new task was successfully acquired after about ten epochs. A lesson we learned here is that it is important to provide sufficient pause time between tasks to avoid unwanted association, a phenomenon well recognized in animal learning.

Although for experiments some particular task examples have to be used, the developmental program is not written for any particular task. For example, after programming, a sensory context can be any short verbal phrase in any language, what verbal phrase is associated with what action is arbitrary, and the number and the length of complex sensorimotor sequence are not fixed. This has not been done before.

\section{EXPERIMENTS ON THE SAIL ROBOT}

SAIL, shown in Fig. 5(I), is a human-size mobile robot housemade at Michigan State University. It has a drive-base, a sixjoint robot arm, a neck, and two pan-tilt units on which two CCD cameras (eyes) are mounted. A wireless microphone functions as an ear. SAIL has four pressure sensors on its torso and 28 touch sensors on its eyes, arm, neck, and bumper. Its main computer is a dual-processor PII PC workstation with $512 \mathrm{MB}$ RAM and an internal 27 GB three-drive disk array. All the sensory information processing, memory recall and update, as well as effector controls were done in real-time.

We have conducted some experiments on the SAIL robot by interacting with it through its auditory sensor, a microphone, 


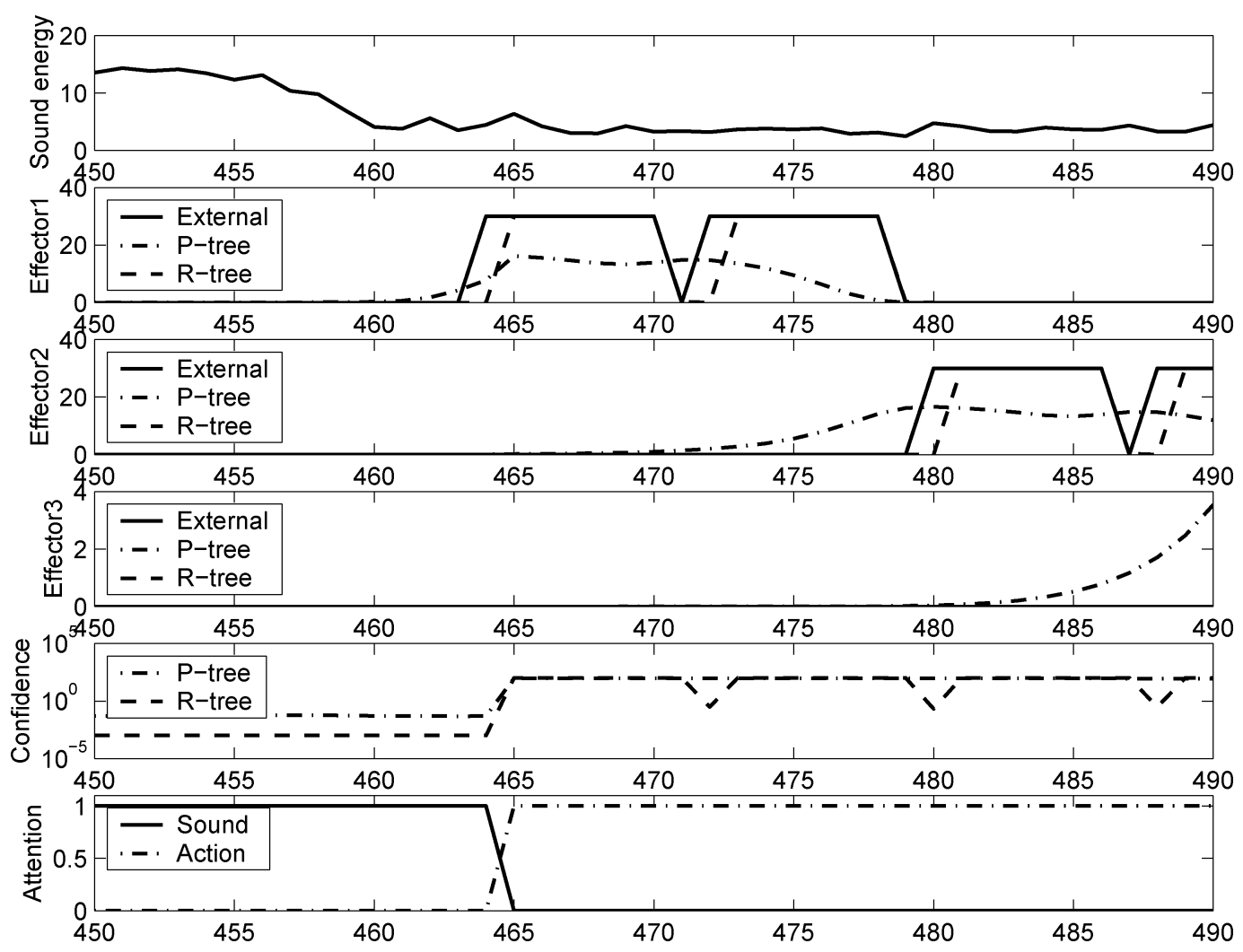

Fig. 20. A closer look at the test session of Step 2).

TABLE I

Performance of AudioDeVeloper Trained By Multiple Users

\begin{tabular}{|c|c|c|c|c|c|c|c|c|}
\hline Epoch No. & 6 & 7 & 8 & 9 & 10 & 11 & 12 & 13 \\
\hline C.R.1 (\%) & 42.86 & 57.14 & 60.32 & 71.43 & 74.60 & 74.60 & 74.60 & 77.78 \\
C.R.2 (\%) & 1.59 & 9.52 & 26.19 & 38.89 & 42.06 & 57.94 & 67.46 & 73.81 \\
\hline Epoch No. & 14 & 15 & 16 & 17 & 18 & 19 & 20 & \\
\hline C.R.1 (\%) & 77.78 & 81.75 & 84.92 & 84.92 & 89.68 & 91.27 & 92.86 & \\
C.R.2 (\%) & 76.98 & 81.75 & 84.92 & 84.92 & 89.68 & 91.27 & 92.86 & \\
\hline
\end{tabular}

and the microswitch sensors on its arm. One of the switch sensors was defined as a dedicated sensor to accept reinforcement signals. Specifically, if the reading of that sensor was positive, the reward was 1 , and if the reading was negative, the reward was -1 . Two other switch sensors were used to impose the four basic arm actions, drawing four petals, as shown in Fig. 5(II).

First, we taught the robot the basic actions in the same way as we did in Section V. Specifically, the training process went as follows: (1) The trainer spoke one of the verbal commands ("one," "two," "three," or "four") in this experiment. (2) At the end of the utterance, the trainer pressed the switch sensor of SAIL to impose the corresponding one of the four petal drawing actions. (3) Wait for 3-4 s and go back to (1). The trainer needed to repeat some of the commands for SAIL to practice. However, according to our observation, within three repeats, a single behavior can be established with the correct rate of $95 \%-100 \%$. This type of fast learning is not possible with iterative mapping learning algorithms such as artificial neural networks (see the discussions in [29] and [41]). However, it is accomplished here thanks to IHDR, which enables fast, one-instance learning. The main reason is that IHDR dynamically creates new parameters in the form of microclusters as explained above, instead of iteratively adjusting a fixed number of parameters.

The internal clusters of IHDR for auditory inputs are not meaningful for visualization (see Fig. 16). Figs. 17-20 are meant to visualize the developmental process. Clusters of visual inputs are more intuitive for visualization [28], [41].

\section{A. Behavior Extinction}

We first report an experiment showing how to "extinct" the established behaviors of the robot. The training process goes as follows: (1) The trainer speaks "one." (2) If the robot moves its arm, the trainer presses a reward switch sensor to give a punishment. (3) Wait for 3-4 s and go back to (1).

To trace the internal changes of the robot, we saved the information related to decision making at every time step at an interval of about $20 \mathrm{~ms}$. The upper panel of Fig. 21(a) shows the sound volume along the time axis. The vertical dash lines show the time instances when the robot started the upper-petal drawing. Among them, the first one is when the trainer imposed the action. The lower panel of Fig. 21(a) shows the changes of $Q$ values of the two actions associated with a particular primitive prototype. A nonaction means the robot stays still and action 1 means upper-petal drawing. In the real-time experiment, there were typically more than 1000 primitive prototypes saved in each IHDR tree. We got hold of the best matched primitive prototype when it was retrieved. For the time instances when a particular primitive prototype was not retrieved, we simply show its $Q$ value when it was retrieved previously. Therefore, the $Q$ value of a particular prototype-action pair changed only when the prototype was visited. This was also the reason why 

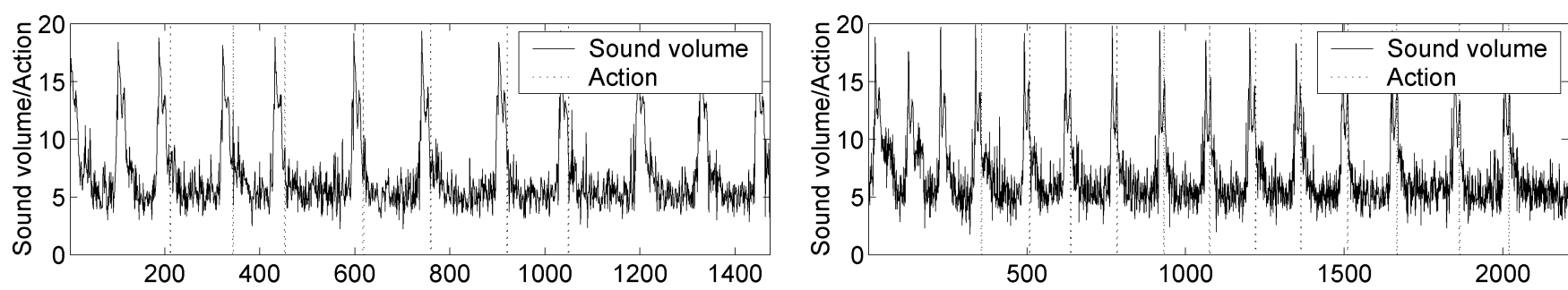

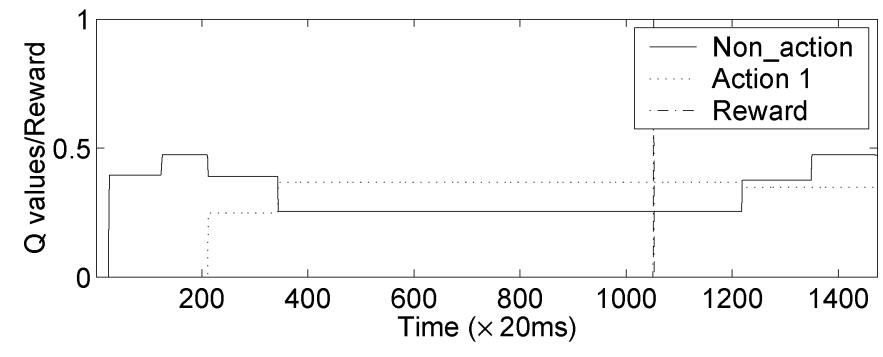

(a)

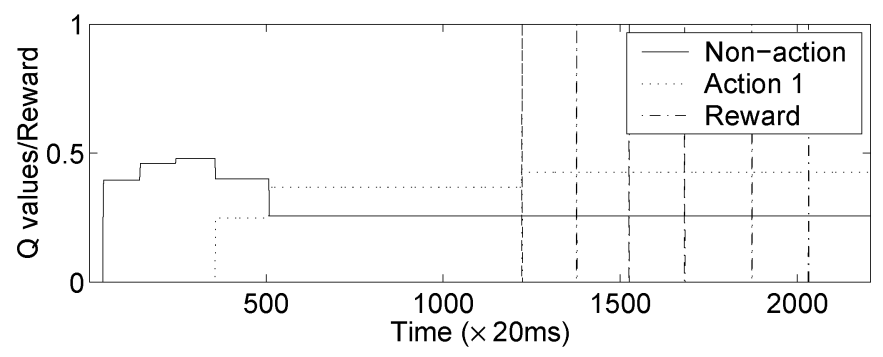

(b)

Fig. 21. (a) The behavior changes of SAIL with the two-level architecture. (b) The behavior changes of SAIL with the one-level architecture.

the changes of the $Q$ values showed up much later than the reinforcement signals. However, we do see the $Q$ value of action 1 starts to grow after the action was imposed. At the same time, that of the nonaction dropped. This means the robot gradually preferred action 1 to the nonaction. After receiving a punishment reinforcement signal at the time instance shown by the vertical dash line in the lower panel of Fig. 21(a), the robot's preference reversed by the changing $Q$ values. From the upper panel, we can see that the previously established behavior was extinct thereafter.

For comparison, we did the same experiment on a single-level system. As shown in Fig. 21(b), although the system can establish the actions, it took it much longer to extinct the established behavior, which shows the power of the multilevel architecture. In animal learning, persistent zero reward can also extinct a learned behavior. The same is true for our computational model (propagating zero rewards) but the extinction process takes longer than the one with negative rewards. According to Pavlov [1], extinction is not simply unlearning what has been learned. For example, conditioning may proceed more rapidly with previously extinguished conditioned stimuli than with novel stimuli [17]-[19]. The memory of previously extinguished conditioned stimuli, similar to what IHDR stores, might help the rapid reacquisition. A higher level process may be also involved, in addition to the sensorimotor process modeled here.

\section{B. Task Transfer}

In this experiment, we taught the robot to do the composite action. The training process goes as follows: (1) A trainer speaks "start," followed by "one," "two," "three," and "four." (2) Wait for 3-4 $\mathrm{s}$ and go back to (1). This is a process where the trainer teaches the system to do the composite action through verbal instructions. After repeating the procedure several times, the trainer speaks only "start." If the robot performs the actions continuously until action $n$, where $n=1,2,3, o r$, we count it as a success up to action $n$. Apparently, when $n=4$, the robot learned the whole new task. Fig. 22 shows that the SAIL robot

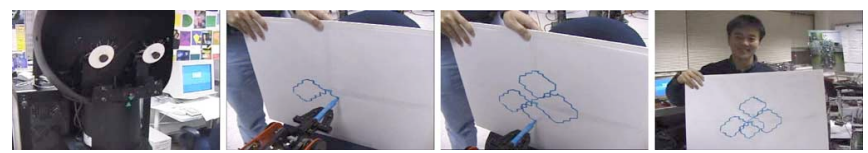

Fig. 22. The SAIL robot learned the new task after verbal instructions by human trainers.

TABLE II

PERFORMANCE OF SAIL DOING TASK TRANSFER

\begin{tabular}{|c|c|c|c|c|}
\hline No. of Actions & 1 & 2 & 3 & 4 \\
\hline C.R. (\%) & 100 & 100 & 95 & 95 \\
\hline
\end{tabular}

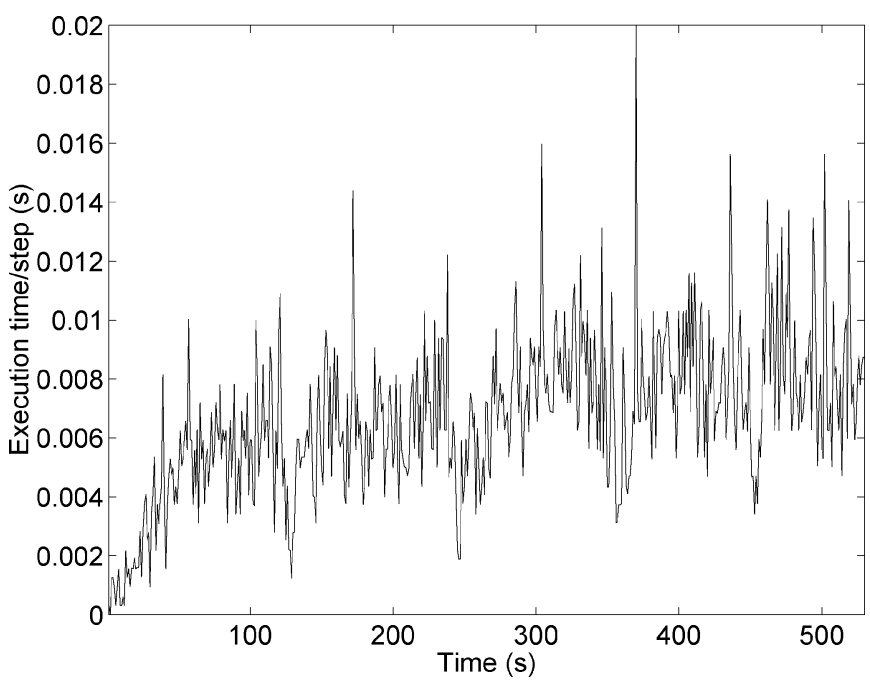

Fig. 23. The average execution time of the two-level system in each execution step is lower than $18.1 \mathrm{~ms}$, the required interval of each speech frame.

learned to draw the whole four-petal flower upon hearing the new command by transferring previously learned skills.

We repeated (20 times) the experiment of teaching SAIL to draw individual petals and the four-petal flower. Each experiment took about 30 to $40 \mathrm{~min}$. Since each petal drawing movement took the robot about 4 to $5 \mathrm{~s}$, the total training time was 

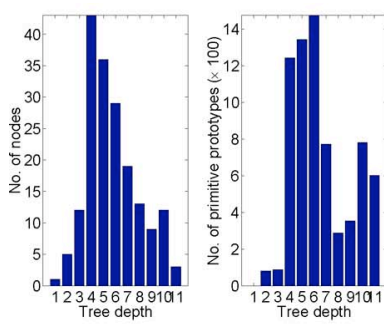

(a)

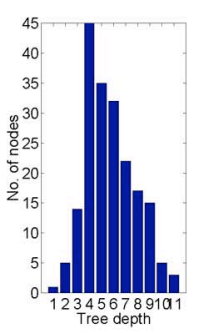

(b)

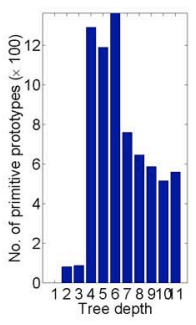

(a) R-tree of the first level LBE. (b) P-tree of the first level LBE. (c) P-tree of the Fig. 24. The node
second level LBE.

relatively long considering the fact that only one speaker was involved. Table II shows that there was one case among 20 when the robot failed to learn the whole new task. In that particular case, the robot confused the command "start" with the command "three" because the onsets of these two words $[s]$ and $[\theta]$ are similar. As a result, the command "start" sometimes elicited action one followed by action two, and sometimes elicited action three.

\section{Execution Time and Memory Size}

The IHDR trees keep clusters (similar to developed neurons), instead of raw samples which are potentially unlimited. Typically, the more complex the sound distribution is, the larger the trees are due to the need to recruit more clusters (neurons), and larger tree sizes mean (slightly) longer retrieval time because the IHDR has a logarithmic time complexity. To push the system to its extremes in terms of tree size, we played loud music and recorded the execution time of above computations within each time step.

Fig. 23 shows that the execution time tended to grow at the beginning and it flattened out after about $100 \mathrm{~s}$, an indication of the logarithmic time complexity. Overall, the execution time of each time cycle is well under the interval of $18.1 \mathrm{~ms}$, though it varies all the time because a primitive prototype may be stored at or retrieved from different depths of the trees. The total size of the three trees reached about $300 \mathrm{MB}$ after $550 \mathrm{~s}$ of "music listening." We do not expect that the time is a major issue for very large IHDR trees because of its logarithmic time complexity. To provide an idea of the structure of the trees, some structure data about the resulted IHDR trees are shown in Fig. 24, where the horizontal axes denote the depth of the tree and the vertical axes are the number of nodes or primitive prototypes saved. The P-tree of $L_{2}$ is significantly smaller than the R-tree and the P-tree of $L_{1}$ because of the "grouping" we mentioned in Section IV.

At the beginning of this paper, we reviewed existing work on classical conditioning or secondary conditioning using a symbolic internal representation. In the open-ended developmental framework here, the human trainer is not allowed to gain access to the internal representation so that similar stimuli are associated with a single (atomic) representation. Therefore, the correspondence between environmental stimuli and internal representation (vector prototypes or neural patterns) are always many-to-many. Further, the developmental mechanism must enable reliable task transfer under not only such many-to-many correspondences, but also the absence of the hint context required by the basic skill. In such real-time online learning, only a few practices are allowed with a very tight time deadline with a very large memory.

The major factors that made this very challenging learning mode successful include: 1) the IHDR which automatically derive hierarchical tree (coarse-to-fine) representation of the most discriminating features to enable generalization among similar prototypes; 2) the double-tree architecture that enables the R-tree to predict immediate context and the P-tree to predict near future; and 3) the multilevel architecture that enables soft abstraction to further enable generalization across similar prototypes in the space of internal representation. If 1) was replaced by a nearest neighbor regressor, not only did performance degrade significantly, but the speed slowed down to a crawl. If 2) or 3) were absent, the task transfer completely failed. In fact, 2) and then 3 ) were added after we experimentally observed a total failure.

Due to the need for avoiding any symbolic representation for open ended development, no existing computational work (including those reviewed earlier), other than the one presented here, is applicable to the challenging 1) real-time, online, incremental acquisition of basic sensorimotor skills and 2) scaffolding to more complex sensorimotor behaviors through transfer of previously learned basic skills to open ended new, but different context settings and task settings. As compared in [28], no other fast enough regressor comes close in performance for the high-dimensional input space here, including CART, C5.0, CO1, and SVM.

\section{CONCLUSION}

The capability of transferring previously acquired sensorimotor skills to new and more complex task settings is crucial for a robot to autonomously learn an open ended number of complex sensorimotor skills that are not known during the programming time. In this report, we presented a theory, principles, an architecture, and the computational framework of a developmental agent capable of task transfer. Our experiments showed that it is theoretically sound and experimentally practical to construct an artificial device that is capable of such task transfer during its open-ended skill development.

The major conceptual contribution of the work presented here is a set of principles that unify the traditional explanation-based human and animal learning types: classical conditioning, instrumental conditioning, and task transfer for sensorimotor skills by 
a single online learning, adaptive architecture. From the context prediction perspective, classical conditioning and instrumental conditioning are special cases of task transfer. The presented computational framework explicitly models the prediction mechanism over both external and internal contexts, and enables the agent to bring the desired context into a close temporal neighborhood for context prediction.

The major technical contributions of the work include the detailed algorithmic design, and software implementation that successfully addresses two major technical challenges, missing context and soft abstraction. Specifically, by extending the backpropagation idea from Q-learning, the P-tree backpropagates not only the primed value but also the primed context, thanks to its numeric representation, in order to realize the near-future priming. At the same time, the R-tree realizes the immediate priming. The intermodal selective attention mechanism allows the agent to choose appropriate inputs to the double-tree mappings. The gating system enables the agent to choose appropriate outputs from R-tree and P-tree. The above mechanisms work together to handle the absence of the hint context.

To address soft abstraction, a multilevel architecture is proposed and implemented. Different levels of sensorimotor systems have different spatial and temporal granularities. The lower level has a fine granularity and captures the details of the wide variation of signals in the real world. The higher level has coarser granularity and enables the generalization across similar prototypes in order to efficiently handle the numerical representation.

These advances are impossible without our prior work that addressed: 1) automatic feature derivation from high-dimensional sensory inputs of context vectors; 2) incremental generation and updates for associative mapping; 3 ) wide variation of stimuli that correspond to the same action output but without use of any internal symbolic representation; and 4) scalability to large memory but still keeping a real-time speed using low cost PC technology. This is the first work to realize task transfer under the open-ended developmental mode. Avoiding the use of any internal symbolic representation is the key to realizing open-ended development.

Probably having an implication to understanding how humans form abstract symbolic concepts from raw numerical sensory inputs, we have demonstrated that symbol-like behaviors, including complex phenomena such as task transfer, can emerge from nonsymbolic distributed vector-type internal representation without using any internal symbolic representation. Symbol-free representation is a must for autonomous development.

This report is an archival journal version of our work started from 2000 [42], [43]. Other interesting speech research studies that were parallel with and closely related to our work include [44] and [45], although fully task-nonspecific incremental openended development was not the objective of their work.

Our future work includes testing the presented architecture on more sensing modalities and more effectors. Some early work on this multimodal learning has been done in [46]. In this paper, we restrict ourselves to task-nonspecific sensorimotor skill development. The extension to high-level task-nonspecific autonomous thinking process has been recently proposed by Weng [47], [48].

\section{REFERENCES}

[1] I. P. Pavlov, Conditioned Reflexes: An Investigation of the Physiological Activity of the Cerebral Cortex. London, U.K.: Oxford Univ. Press, 1927.

[2] N. J. Mackintosh, Conditioning and Associative Learning. London, U.K.: Oxford Univ. Press, 1983.

[3] R. S. Sutton and A. G. Barto, "Toward a modern theory of adaptive networks: Expectation and prediction," Psychol. Rev., vol. 88, pp. 135-170, 1981.

[4] - "A temporal-difference model of classical conditioning," in Proc. 9th Conf. Cogn. Sci. Soc., 1987, pp. 355-378.

[5] A. H. Kloph, "A neuronal model of classical conditioning," Psychobiology, vol. 16, pp. 85-125, 1988

[6] C. Balkenius, "Generalization in instrumental learning," in Proc. 4th Int. Conf. Simulation of Adaptive Behavior, From Animals to Animats 4, P. Maes, M. J. Mataric, and J. A. Meyer, Eds., MIT Press, Cambridge, MA, 1996.

[7] C. Balkenius and J. Moren, "Computational models of classical conditioning: A comparative study," in Proc. 5th Int. Conf. Adaptive Behavior, From Animals to Animats 5, R. Pfeifer, B. Blumberg, J. A. Meyer, and S. W. Wilson, Eds., 1998, pp. 348-353.

[8] D. S. Touretzky and L. M. Saksida, "Operant conditioning in skinnerbots," Adaptive Behav., vol. 5, no. 3/4, pp. 219-247, 1997.

[9] L. M. Saksida, S. M. Raymond, and D. S. Touretzky, "Shaping robot behavior using principles from instrumental conditioning," Adaptive Behav., vol. 22, no. 3/4, pp. 231-249, 1998.

[10] O. Sporns, N. Almassy, and G. M. Edelman, "Plasticity in value systems and its role in adaptive behavior," Adaptive Behav., vol. 8, no. 2, pp. 129-148, 2000 .

[11] J. Weng, J. McClelland, A. Pentland, O. Sporns, I. Stockman, M. Sur and E. Thelen, "Autonomous mental development by robots and animals," Science, vol. 291, no. 5504, pp. 599-600, 2001.

[12] J. Weng and I. Stockman, "Autonomous mental development: Workshop on development and learning," AI Mag., vol. 23, no. 2, pp. 95-98, 2002.

[13] M. Domjan, The Principles of Learning and Behavior, 4th ed. Belmont, CA: Brooks/Cole, 1998.

[14] B. Schwartz and S. J. Robbins, Psychology of Learning and Behavior. New York: Norton, 1994.

[15] C. L. Hull, "Knowledge and purpose as habit mechanisms," Psychol. Rev., vol. 30, pp. 511-525, 1930.

[16] _ "Goal attraction and directing ideas conceived as habit phenomena," Psychol. Rev., vol. 38, pp. 487-506, 1931.

[17] J. Konorski and G. Szwejkowska, "Chronic extinction and restoreation of conditioned reflexes: I. Extinction against the excitatory background," Acta Biologiae Experimentalis, vol. 15, pp. $155-170,1950$.

[18] _ "Chronic extinction and restoration of conditioned reflexes: Iv. The dependence of the course of extinction and restoration of conditioned reflexes on the "history" of the conditioned stimulus (the principle of the primacy of first training)," Acta Biologiae Experimentalis, vol. 16, pp. 95-113, 1951.

[19] R. M. Napier, M. Macrae, and E. J. Kehoe, "Rapid reacquisition in conditioning of the rabbit's nictitating membrane response," $J$. Experimental Psychology: Animal Behav., vol. 18, pp. 182-192, 1992.

[20] R. A. Rescorla and A. R. Wagner, "A theory of Pavlovian conditioning: Variations in the effectiveness of reinforcement and nonreinforcement," in Classical Conditioning II: Current Research and Theory, A. H. Black and W. F. Prokasy, Eds. New York: Appleton-Century-Crofts, 1972, pp. 64-99.

[21] Y. Zhang, J. Weng, and W. Hwang, "Auditory learning: A developmental method," IEEE Trans. Neural Netw., vol. 16, no. 3, pp. 601-616, May 2005.

[22] J. P. Byrnes, Cognitive Development and Learning in Instructional Contexts. Boston, MA: Allyn and Bacon, 1995.

[23] L. S. Vygotsky, Thought and Language. Cambridge, MA: MIT Press, 1962, trans. E. Hanfmann \& G. Vakar.

[24] D. J. Wood, J. S. Bruner, and G. Ross, "The role of tutoring in problemsolving," J. Child Psychology Psychiatry, pp. 89-100, 1976.

[25] J. Weng, "Developmental robotics: Theory and experiments," Int. J. Humanoid Robotics, vol. 1, no. 2, pp. 199-235, 2004.

[26] _ _ "A theory for mentally developing robots," in Proc. IEEE 2nd Int. Conf. Development Learn., Cambridge, MA, Jun. 12-15, 2002, pp. 131-140. 
[27] J. Weng and S. Zeng, "A theory of developmental mental architecture and the Dav architecture design," Int. J. Humanoid Robotics, vol. 2, no. 2, pp. 145-179, 2005.

[28] W. S. Hwang and J. Weng, "Hierarchical discriminant regression," IEEE Trans. Pattern Anal. Mach. Intell., vol. 22, no. 11, pp. 1277-1293, Nov. 2000.

[29] J. Weng and W. Hwang, "Online image classification using IHDR," Int. J. Document Anal. Recogn., vol. 5, no. 2-3, pp. 118-125, 2002.

[30] C. Watkins and P. Dayan, "Q-learning," Mach. Learn., vol. 8, pp. 279-292, 1992.

[31] X. Huang and J. Weng, "Value system development for a robot," in Proc. Int. Joint Conf. Neural Netw., Jul. 26-29, 2004, pp. 2883-2888.

[32] D. R. Cox, "Statistical analysis of time series: Some recent developments," Scand. J. Statist., vol. 8, no. 2, pp. 93-115, 1981.

[33] S. L. Zeger and B. Qaqish, "Markov regression models for time series: A quasi-likelihood approach," Biometrics, vol. 44, no. 4, pp. 1019-1031, Dec. 1988.

[34] J. Weng, W. S. Hwang, Y. Zhang, C. Yang, and R. Smith, "Developmental humanoids: Humanoids that develop skills automatically," in Proc. 1st IEEE Conf. Humanoid Robots, Cambridge, MA, Sep. 7-8, 2000, CD-ROM.

[35] Y. Zhang and J. Weng, "Grounded auditory development by a developmental robot," in Proc. IEEE Int. Joint Conf. Neural Netw., Washington, DC, Jul. 14-19, 2001, pp. 1059-1064.

[36] R. A. Brooks, "A robust layered control system for a mobile robot," IEEE J. Robotics Autom., vol. 2, no. 1, pp. 14-23, Mar. 1986.

[37] R. S. Sutton and A. Barto, Reinforcement Learning. Cambridge, MA: MIT Press, 1998.

[38] E. R. Kandel, J. H. Schwartz, and T. M. Jessell, Eds., Principles of Neural Science, 3rd ed. Norwalk, CT: Appleton \& Lange, 1991.

[39] J. R. Deller, Jr., J. G. Proakis, and J. H. L. Hansen, Discrete-Time Processing of Speech Signals. New York: Macmillan, 1993.

[40] J. Weng, Y. Zhang, and W. S. Hwang, "Teaching a learning vehicle A developmental perspective," in Proc. Robotics Mechatronics Congr. Singapore, Jun. 6-8, 2001, CD-ROM.

[41] J. Weng and W. S. Hwang, "Incremental hierarchical discriminant regression," IEEE Trans. Neural Netw., 2007, accepted for publication.

[42] Y. Zhang and J. Weng, "Chained action learning through real-time interactions," in Proc. IEEE Int. Joint Conf. Neural Netw., Honolulu, HI, May 12-17, 2002, pp. 2012-2-17.

[43] _ , "Action chaining by a developmental robot with a value system," in Proc. IEEE 2nd Int. Conf. Development Learn., Cambridge, MA, Jun. 12-15, 2002, pp. 53-60.

[44] Q. Liu, S. Levinson, Y. Wu, and T. Huang, "Robot speech learning via entropy guided LVQ and memory association," in Proc. IEEE Int. Joint Conf. Neural Netw., Washington, DC, Jul. 14-19, 2001, pp. 2176-2181.

[45] D. Roy and A. Pentland, "Learning words from sights and sounds: A computational model," Cogn. Sci., vol. 26, no. 1, pp. 113-146, 2002.

[46] Y. Zhang and J. Weng, "Conjunctive visual and auditory development via real-time dialogue," in Proc. 3rd Int. Workshop Epigenetic Robotics, Boston, MA, Aug. 4-5, 2003, pp. 974-980.

[47] J. Weng, "A theory of developmental architecture," in Proc. 3rd Int. Conf. Development Learn., La Jolla, CA, Oct. 20-22, 2004, CD-ROM.
[48] — - "On developmental mental architectures," Neurocomputing, 2007, accepted for publication.

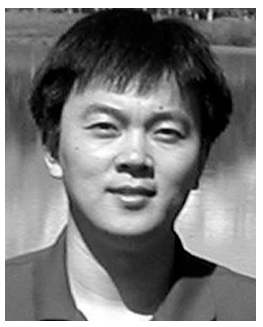

Yilu Zhang (M'02) received the B.S. and M.S. degrees in electrical engineering from Zhejiang University, Zhejiang, China, in 1994 and 1997, respectively, and the Ph.D. degree in computer science from Michigan State University, East Lansing, in 2002.

He joined the Research and Development Center of General Motors Corporation, in 2002, and currently holds a position as Senior Researcher. His research interests include human machine interactions, machine learning, speech/image processing, data mining, and their applications in the vehicle

technology domain.

Dr. Zhang is a member of SAE, Sigma Xi International Honor Society, and the Autonomous Mental Development Technical Committee of the IEEE Computational Intelligence Society. He serves as an Associate Editor of the International Journal of Humanoid Robotics

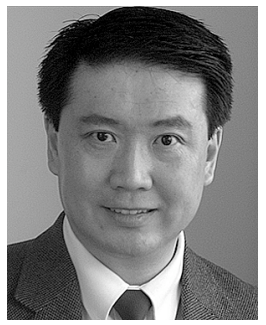

Juyang (John) Weng (', $\left.85-\mathrm{M}^{\prime} 89-\mathrm{SM}^{\prime} 05\right)$ is currently a Professor at the Department of Computer Science and Engineering, Michigan State University, East Lansing. He initiated and supervised the SAIL (Self-Organizing Autonomous Incremental Learner) and Dav projects, in which he and his coworkers have designed and custom built their SAIL and Dav robots for research on robotic computational realization of autonomous mental development. More detail is available on line at http://www.cse.msu.edu/ weng/. $\mathrm{He}$ is the author or coauthor of over 180 reviewed research articles and book chapters published in books, journals, conferences, and workshops. His research interests include mental architectures, computational neuroscience, mental development, biologically inspired neural systems, vision, audition, touch, human-machine multimodal interface, and intelligent robots.

Dr. Weng is an Associate Editor of the IEEE TRANSACTIONS ON PATTERN RECOGNITION AND MACHINE INTELLIGENCE and an Associate Editor of the IEEE TRansactions ON IMAge PROCESSING. He is an Editor-in-Chief of the International Journal of Humanoid Robotics and the founding Chairman of the Governing Board of the multidisciplinary International Conferences on Development and Learning (ICDL) (http://cogsci.ucsd.edu/ triesch/icdl/). He was the founding Chairman of the Autonomous Mental Development Technical Committee of the IEEE Computational Intelligence Society (2004-2005). He was Program Chairman of the NSF/DARPA Workshop on Development and Learning (WDL) held April 2000 at Michigan State University (MSU) (http://www.cse.msu.edu/dl/), and Program Chairman of the International Conference on Development and Learning 2002 (ICDL'02), held at the Massachusetts Institute of Technology, Cambridge, MA, June 2002 (http://www.egr.msu.edu/icd102/). 